
Applied and
Computational
Mathematics
Division

\title{
MODULATION OF FOSSIL FUEL PRODUCTION BY GLOBAL TEMPERATURE VARIATIONS, II.
}

Bert W. Rust and Frank J. Crosby

January 1994

\section{U.S. DEPARTMENT OF COMMERCE \\ National Institute of Standards and Technology \\ Gaithersburg, MD 20899}





\section{Modulation of Fossil Fuel \\ Production by Global \\ Temperature Variations, II.}

Bert W. Rust
Frank J. Crosby

U.S. DEPARTMENT OF COMMERCE

Technology Administration

National Institute of Standards

and Technology

Computing and Applied Mathematics Laboratory Applied and Computational Mathematics Division Gaithersburg, MD 20899

January 1994

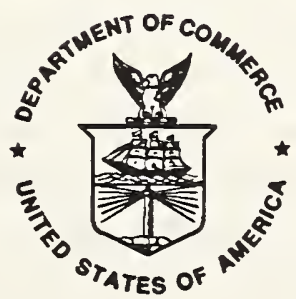

U.S. DEPARTMENT OF COMMERCE Ronald H. Brown, Secretary

TECHNOLOGY ADMINISTRATION

Mary L Good, Under Secretary for Technology

NATIONAL INSTITUTE OF STANDARDS

AND TECHNOLOGY

Arat Prabhakar, Director 



\title{
MODULATION OF FOSSIL FUEL PRODUCTION BY GLOBAL TEMPERATURE VARIATIONS, II.
}

\author{
Bert W. Rust and Frank J. Crosby \\ Applied and Computational Mathematics Division \\ Building 101, Room A-238 \\ National Institute of Standards and Technology \\ Gaithersburg, MD 20899
}

\begin{abstract}
We extend the earlier work of Rust and Kirk (1982) on the inverse modulation of global fossil fuel production by variations in Northern Hemispheric temperatures. The present study incorporates recent revisions and extensions of the fuel production record and uses a much improved temperature record. We show that the new data are consistent with the predictions of the original Rust-Kirk model which we then extend to allow for time lags between variations in the temperature and the corresponding responses in fuel production. The modulation enters the new model through the convolution of a lagged averaging function with the temperature time-series. We also include explicit terms to account for the perturbations caused by the Great Depression and World War II. The final model accounts for $99.84 \%$ of the total variance in the production record. The temperature modulation produces variations of as much as $30 \%$ in the total production. This modulation represents a feedback which is consistent with the predictions of the Gaia hypothesis for a planetary greenhouse temperature control. We use the new model to calculate 20-year fuel production predictions for three temperature scenarios which hopefully bracket the possibilities for temperature behavior during that time.
\end{abstract}





\section{Introduction}

A previous analysis of the historical record of global fossil fuel production has suggested that the basically exponential growth is modulated inversely by variations in the average surface temperature of the Northern Hemisphere [Rust and Kirk (1982)]. Since that study was published, greatly improved temperatures records have become available, and the global production record has been updated and extended by several years. In this paper, we first repeat the original analysis with the new data and then generalize the model to allow for time lags between the temperature variations and their corresponding responses in the production record.

Throughout this paper we will use the term "Paper I" to refer to the original Rust and Kirk study which we briefly review in Section 2, where we also show that the addition of the temperature modulation term to the exponential model produces a statistically significant reduction in the residual variance. In Section 3 we examine a new temperature record compiled by Jones, et al (1986, $1988,1992)$ and a revised and extended record of production data compiled by Marland $(1989,1992)$. The new additions to the data are consistent with the predictions of the Rust-Kirk model, and the addition of the temperature modulation to the exponential model again produces a significant reduction of the residual variance. In Section 4, still further reductions are obtained by allowing for a time lag between the temperature variations and their responses in the production record. The length of the time lag becomes an additional free parameter in the model, but its inclusion produces a statistically significant reduction in the residual variance. In Section 5 we replace the assumption of a single fixed lag for the response with the assumption that production depends on the average temperature in some fixed time interval immediately preceding the current time. This produces an additional reduction in the residual variance without increasing the number of free parameters in the model.

The temperature averaging used in Section 5 corresponds to a convolution of the temperature record with a simple boxcar averaging function. In Section 6 we show that this boxcar model is based on the same simple linear dynamics as the Rust-Kirk model. Even though it gives a much better fit to the data than an unmodulated exponential, it still does not completely capture the temperature modulation. We show that the reason for this failure is the two precipitous drops in production caused by the Great Depression and World War II. In Section 7 we include these two drops as explicit innovations in the model, introducing two additional free parameters in the process. This increases the total to six, so we perform extensive testing to establish their 
statistical significance. Not only do the new terms produce a significant reduction in the residual variance, but they also free the modulation term to completely capture the temperature effect.

In Section 8 we introduce a more general temperature averaging function constrained only to be nonnegative and to subtend unit area on a finite time domain. The boxcar function is a simple example which depends on a single free parameter specifying the length of the memory. Another one-parameter example is a triangular function, but it gives a sum of squared residuals almost identical to that obtained with the boxcar. Further reductions in the residual variance require additional free parameters defining the shape of the averaging function. In Section 9 we add two more parameters to specify a quadrilateral function which produces a statistically significant reduction.

In Section 10 we develop a transfer function for the modulation, using an annual mesh for the transfer function ordinates. Each ordinate becomes a free parameter in the model. We determine the length of the transfer filter by requiring each ordinate to produce a significant reduction of the residual variance. The final result is a model with 13 free parameters which gives a fit that acounts for $99.85 \%$ of the total variance in the production record. In Section 11 we employ a Monte Carlo study to demonstrate that this impressive fit is not simply attributable to the large number of free parameters, but depends crucially on the use of the temperature time-series as the modulating process.

In Section 12 we reduce the number of free parameters in the model by replacing the discrete transfer function ordinates with a continuous function obtained by fitting an optimal smoothing spline to them. This reduces the number to 5 , which is the same as the original Rust-Kirk model when the latter is augmented with terms for the Depression and World War II. The model explains $99.84 \%$ of the total variance. We also isolate that part of the total variation attributable to the temperature modulation and show that the magnitude of that variation exceeds the combined magnitudes of the perturbations caused by the Depression and the War.

In Section 13 we briefly review the Gaia Hypothesis and point out that it would predict an inverse temperature modulation of fossil fuel production if a greenhouse warming were initiated by releases of fossil fuel $\mathrm{CO}_{2}$ to the atmosphere. In Section 14 we conclude by calculating 20 -year predictions of total fossil fuel production for three possible future temperature scenarios. 


\section{The Rust-Kirk Model}

In Paper I, Rust and Kirk (1982) showed that global fossil fuel production for the years 1870-1974 exhibited exponential growth with a rate modulated inversely by temperature variations in the Northern Hemisphere. Using linear dynamics, they developed a simple stockpile model for fossil fuel production and consumption which gave a recursion relation for the annual production. If $P(t)$ and $P(t-1)$ are predicted values for global production in years $t$ and $(t-1)$, then the recursion can be written

$$
P(t)=\{[1+a]-b[T(t)-T(t-1)]\} P(t-1),
$$

where $T(t)$ and $T(t-1)$ are the corresponding yearly average temperatures for the Northern Hemisphere, and $a, b$, and $P_{0}=P(0)$ are parameters determined by least squares fitting. When the time step shrinks to zero, this recursion is replaced by the analogous differential equation

$$
\frac{d P}{d t}=\left(\alpha-\beta \frac{d T}{d t}\right) P
$$

which has the solution

$$
P(t)=P_{0} \exp \{\alpha t-\beta[T(t)-T(0)]\},
$$

with free parameters $\alpha, \beta$, and $P_{0}$. Throughout this paper we shall use epoch 1870.0 as the zero for the time scale, though we shall usually show the data and fits plotted against the year rather than the time $t$ used in making the fits.

For $T(t)$, Rust and Kirk used a smoothing spline approximation to the record of 5-year average temperatures compiled by Mitchell (1961) and Brinkman (1976). These data and the spline representation are shown in Figure 1. The least squares fit of (2.3) to the production record is shown as a solid curve in Figure 2 which also shows a dashed curve representing the fit of an unmodulated exponential,

$$
P(t)=P_{0} \exp (\alpha t) .
$$

The estimates of the adjustable parameters for the two fits, together with their estimated $\pm 1 \sigma$ uncertainties, are given in Table 1 . The column labelled SSR gives the sum of squared residuals for the fits, i.e.,

$$
\mathrm{SSR}=\sum_{i=1}^{m}\left[P_{i}-P\left(t_{i}\right)\right]^{2},
$$


where $m$ is the number of measured data values $P_{i}$, and $P\left(t_{i}\right)$ is the model prediction at time $t_{i}$. The final column (labelled " $R^{2}$ ") gives the coefficient of determination for each fit, i.e.,

$$
R^{2}=1-\frac{\mathrm{SSR}}{\mathrm{CTSS}},
$$

where CTSS is the corrected total sum of squares. This latter quantity is defined by

$$
\mathrm{CTSS}=\sum_{i=1}^{m}\left[P_{i}-\bar{P}\right]^{2},
$$

where $\bar{P}$ is the average value of the measured data. Thus $R^{2}$ estimates the fraction of the total variance that is explained by the fitted model.

For all the fits described in this paper, we used the interactive nonlinear least squares code INVAR (Wolfe et al, 1987) which is based on the variable separable subroutine VARPRO (Golub and Pereyra, 1973). We used equal weighting which means that, by virtue of their magnitudes, the later data points were weighted more heavily than the earlier ones. This weighting was appropriate because the relative uncertainties in the later production estimates are much smaller than those in the earlier ones.

It is obvious from comparing the two curves in Fig. 2 that adding the tempreature modulation term to the model produced a significant improvement in the fit. Nevertheless we will perform an F-test for significance on the new parameter $\beta$. This same procedure will be repeated in the following each time a new parameter is added to the model. The test compares the SSR for the full model (2.3) with the SSR for the reduced model (2.4) to determine whether or not the $\beta$ term produced a statistically significant reduction in the residual variance. It tests the null hypothesis

$$
\mathcal{H}_{0}: \beta=0 \text {. }
$$

It is based on the statistic

$$
u=\frac{\frac{\rho_{1}-\rho}{n_{h}}}{\frac{\rho}{m-n}}=\left(\frac{\rho_{1}}{\rho}-1\right) \frac{m-n}{n_{h}},
$$

where $\rho$ and $\rho_{1}$ are respectively the SSR values for the full and reduced models, $n$ is the number of free parameters in the full model, and $n_{h}$ is the number of parameters in the null hypothesis. This statistic follows an F-distribution with $n_{h}$ and $m-n$ degrees of freedom. The test procedure is to pick a probability level $p$ and compare the calculated value of $u$ with the percentage point 
$F_{p}\left(n_{h}, m-n\right)$. If $u>F_{p}\left(n_{h}, m-n\right)$, then the null hypothesis is rejected and the value of $\beta$ is significant at the $100 p \%$ level. For the present problem, $m=105, n=3$, and $n_{h}=1$, so

$$
u=\left(\frac{4.454 \times 10^{6}}{7.822 \times 10^{5}}-1\right) \times 103=478.8 .
$$

The usual choice for the probability is $p=0.95$ which gives $F_{p}(1,102)=3.93$. This is much smaller than the calculated $u$, so the null hypothesis is rejected. In fact, $\mathcal{H}_{0}$ is rejected even at the very high probability $p=0.9999$, which gives $F_{p}(1,102)=16.41$.

\section{The New Data}

Since the publication of Paper I, several improved historical temperature records have been compiled. The time-series of annual average Northern Hemisphere, land-based temperatures compiled by Jones et al (1986) and updated and extended by Jones $(1988,1992)$ are shown in Fig. 3 together with a cubic interpolating spline and an optimal smoothing spline fit. Both of these splines will be used in the following to represent the temperature function $T(t)$. To get the smoothing spline, we used the algorithm CUBGCV (Hutchinson, 1986) which chooses the smoothing constant to minimize the generalized cross validation statistic (Craven and Wahba, 1979). In adopting this procedure, we were not making a judgement about the quality of the data. We needed a smoothed representation of it, and minimizing the cross validation statistic provided an objective way for choosing the amount of smoothing.

The fossil fuel production record has also been improved since the publication of Paper I. Marland $(1989,1992)$ has revised the estimates for 1950-1974 and extended the record through 1990 [see also Marland and Boden (1992)]. This new record is plotted in Figure 4 where the dashed curve was obtained by fitting the unmodulated exponential (2.4), and the solid line by fitting the Rust-Kirk model (2.3), using the smoothing spline representation of $T(t)$. The parameter values for these fits are given in Table 2.

The temperature modulation produces a visible improvement in the fit. To determine whether or not this improvement is statistically significant, we again performed the F-test described in Section 2, using the same null-hypothesis (2.8). In the present case, $m=121, n=3, n_{h}=1$, and the $u$-statistic (2.9) becomes

$$
u=\left(\frac{7.324 \times 10^{6}}{3.227 \times 10^{6}}-1\right) \times 118=149.8 \text {. }
$$


Choosing probability $p=0.9999$ gives $F_{p}(1,118)=16.21$ which is much smaller than the value of $u$, so the null hypothesis is resoundingly rejected, and the significance of the temperature modulation is established at the $99.99 \%$ level.

Two features of the data which were not captured by either model are the precipitous drops in production which occurred in 1929-1932, corresponding to the early years of the Great Depression, and in 1944-1945, the final year of World War II. In the first case, production dropped from 1171.9 megatons in 1929 to 873.8 megatons in 1932 . This is a decline of $25 \%$ in only three years. In the second case the production dropped from 1352.2 in 1944 to 1203.6 megatons in 1945, a decline of $11 \%$ in a single year. It is interesting that both of these calamitous events occurred during a period of rising temperatures, but it is clear from Fig. 4 that the Rust-Kirk model does no better than the exponential in accounting for them. We shall see in the following that it is necessary to include these drops as explicit innovations in the model in order to completely isolate and capture the temperature modulation.

\section{A Lagged Response Model}

The inclusion of the temperature modulation produced a significant increase in the variance explained by the model, but an even better fit can be obtained by allowing a time lag in the response to the temperature variations. This lagged response model has the form

$$
\frac{d P}{d t}=\left(\alpha-\beta \frac{d}{d t} T(t-\tau)\right) P
$$

where $\tau$ is a lag parameter which is to be determined by fitting. The solution of this differential equation is

$$
P(t)=P_{0} \exp \{\alpha t-\beta[T(t-\tau)-T(-\tau)]\} .
$$

Fitting this expression gave the parameter values in the last row of Table 2 and the solid curve in Figure 5 which also shows the fit of an unmodulated exponential. Comparing Figure 5 with 4 reveals that the time delay produces a visible improvement in the fit. The statistical significance of that improvement was verified by using the F-test with the null-hypothesis

$$
\mathcal{H}_{0}: \tau=0 \text {. }
$$


Using $m=121, n=4, n_{h}=1$, and the SSR values from Table 2 gives

$$
u=\left(\frac{3.227 \times 10^{6}}{1.651 \times 10^{6}}-1\right) \times 117=111.7
$$

Using probability $p=0.9999$ gives $F_{p}(1,117)=16.22$ which is much smaller than the $u$-value so the null hypothesis is rejected, and the improvement due to the time lag is significant at a level greater than $99.99 \%$.

\section{A Lagged Interval Response Model}

While it is reasonable to expect time delays in the temperature effect, the lagged response model (4.1) is unrealistic because it posits a dependence on the instantaneous temperature at an exact moment $\tau$ years in the past. It is more reasonable to expect a dependence on the whole temperature history in some immediately preceding time interval. The simplest such model has the form

$$
\frac{d P}{d t}=\left(\alpha-\beta \frac{T(t)-T(t-\tau)}{\tau}\right) P,
$$

where $[T(t)-T(t-\tau)] / \tau$ is the average $d T / d t$ on the interval $[(t-\tau), t]$. This differential equation has the solution

$$
P(t)=P_{0} \exp \left\{\alpha t-\beta\left[\frac{1}{\tau} \int_{0}^{t} T\left(t^{\prime}\right) d t^{\prime}-\frac{1}{\tau} \int_{0}^{t} T\left(t^{\prime}-\tau\right) d t^{\prime}\right]\right\},
$$

which, since

$$
\int_{0}^{t} T\left(t^{\prime}-\tau\right) d t^{\prime}=\int_{-\tau}^{0} T\left(t^{\prime}\right) d t^{\prime}+\int_{0}^{t-\tau} T\left(t^{\prime}\right) d t^{\prime}
$$

can also be written

$$
P(t)=P_{0} \exp \left\{\alpha t-\beta\left[\frac{1}{\tau} \int_{t-\tau}^{t} T\left(t^{\prime}\right) d t^{\prime}-\frac{1}{\tau} \int_{-\tau}^{0} T\left(t^{\prime}\right) d t^{\prime}\right]\right\} .
$$

For any value of $\tau$, the indicated integrals can be evaluated by numerically integrating the spline function $T(t)$. For this purpose, we used the adaptive quadrature algorithm Q1DB (Kahaner et al, 1989, Chapt. 5) from the GAMS library (Boisvert et al, 1984).

Using the smoothing spline for $T(t)$ to fit (5.4) gave the parameter estimates in Table 3 . The fitted curve was graphically identical to the solid curve 
in Fig. 5. This is not surprising since the Mean Value Theorem guarantees that there is some point $\left(t-\tau^{\prime}\right)$ on the interval $[(t-\tau), t]$ such that

$$
\frac{d}{d t} T\left(t-\tau^{\prime}\right)=\frac{T(t)-T(t-\tau)}{\tau} .
$$

Thus, if we take $\tau^{\prime}=5.6 \mathrm{yr}$ in model (4.1), then it becomes equivalent to model (5.1) with $\tau=12.0 \mathrm{yr}$.

The 12 year averaging interval was long enough to suggest replacing the smoothing spline representation for $T(t)$ with the interpolating spline, shown as the dotted line in Fig. 3. Although the latter tracks the strong year-to-year temperature variations, the long time-length of the averaging function smooths those variations enough to give the fit shown in Fig. 6, with the parameter values given in the last row of Table 3 . Although the fitted curve exhibits small local wiggles, the SSR and $R^{2}$ values are better than those for the smoothing spline, and the curve does a better job of tracking the dip in production during the last decade. Thus, the interpolating spline improves the quality of the fit without introducing any new free parameters. Unless otherwise noted, we will use the interpolating spline representation of $T(t)$ for all of the remaining fits to be described in this paper.

\section{The Boxcar Averaging Model}

The lagged interval response model (5.4) can also be written

$$
\begin{aligned}
P(t)=P_{0} \exp \left\{\alpha t-\beta\left[\int_{t-\tau}^{t} b\left(t^{\prime}-t ; \tau\right) T\left(t^{\prime}\right) d t^{\prime}\right.\right. \\
\\
\left.\left.\quad-\int_{-\tau}^{0} b\left(t^{\prime} ; \tau\right) T\left(t^{\prime}\right) d t^{\prime}\right]\right\}
\end{aligned}
$$

where

$$
b\left(t^{\prime \prime} ; \tau\right)=\left\{\begin{array}{cl}
\frac{1}{\tau}, & \text { if }-\tau<t^{\prime \prime} \leq 0, \\
0, & \text { otherwise } .
\end{array}\right.
$$

The function $b\left(t^{\prime \prime} ; \tau\right)$ is sometimes called a boxcar averaging function, and we will refer to the model (6.1), or (5.4), as the boxcar model. This way of writing it might appear to be unnecessarily complicated, but it serves as a prototype for the more realistic tapered averaging models that will be introduced in Section 8.

The boxcar model can also be written as a differential equation,

$$
\frac{d P}{d t}=\left\{\alpha-\beta \frac{d}{d t}\left[\int_{t-\tau}^{t} b\left(t^{\prime}-t ; \tau\right) T\left(t^{\prime}\right) d t^{\prime}\right]\right\} P
$$


which makes the underlying assumptions more obvious. These assumptions are essentially the same as those in Paper I, but here instantaneous derivatives replace yearly differences, and the temperature dependence extends for more than a year. We assume that

$$
\frac{d P}{d t}=\frac{d I}{d t}+\frac{d H}{d t}
$$

with $I(t)$ independent of temperature, but

$$
H(t) \propto-\int_{t-\tau}^{t} b\left(t^{\prime}-t ; \tau\right) T\left(t^{\prime}\right) d t^{\prime},
$$

where the minus sign indicates an inverse dependence of $H(t)$ on the temperature variations. Both $d I / d t$ and $d H / d t$ are assumed to vary linearly with global economic activity which is itself assumed to vary linearly with $P(t)$. Thus,

$$
\frac{d I}{d t}=\alpha P(t)
$$

and

$$
\frac{d H}{d t}=-\beta P(t) \frac{d}{d t}\left[\int_{t-\tau}^{t} b\left(t^{\prime}-t ; \tau\right) T\left(t^{\prime}\right) d \dot{i}^{\prime}\right],
$$

where $\alpha$ and $\beta$ are constants which become free parameters in the fits.

The residuals for the boxcar fit, which are plotted in Figure 7, indicate that the model does not completely account for the inverse temperature variations. They exhibit a systematic decline in the years 1910-1945, when tempreatures were rising, and a discernible increase in the years 1945-1970, when temperatures were decreasing. Thus the model has not accounted for all of the temperature modulation. The cause for this shortcoming is the presence of the two sharp drops in production during the years 1929-1932 and 19441945. These are short-term phenomena which are apparently independent of the longer-term temperature variations. In trying to adjust the values of the free parameters to accommodate these two drastic changes, the fit fails to capture all of the temperature variation that the model was designed to describe. In the next section we shall include these two effects as explicit innovations in the model.

\section{Modelling the Depression and the War}

Between 1929 and 1932, fossil fuel production fell by roughly 100 megatons per year, but then recovered almost exactly half of those losses in the subsequent 
3 years. To model these changes, we used an expression of the form

$$
\Delta_{G D}[P(t)]=\gamma D(t)
$$

where $\gamma$ is a non-negative parameter to be determined by fitting, and $D(t)$ is a notch function defined by

$$
D(t)= \begin{cases}0 & , t \leq t_{1929} \\ -\left(t-t_{1929}\right) & , t_{1929}<t \leq t_{1932} \\ -3+0.5\left(t-t_{1932}\right) & , t_{1932}<t \leq t_{1935} \\ -1.5 & , t_{1935}<t\end{cases}
$$

with $t_{1929}, t_{1932}$, and $t_{1935}$ being the times corresponding to years 1929,1932 , and 1935 respectively. We assumed that the term (7.1) is a addition to the model rather than a replacement for it during years 1929-1935. We initially tried using only the drop section of the notch, i.e., years 1929-1932, but found that the resulting model could account for only about half of the decrease in those years. It seems reasonable to suppose that a significant fraction of the decrease was caused by financial dislocations rather than by destruction or shrinking of the production infrastructure. The partial recovery in the next 3 years might then be attributed to a resolution of some of those financial difficulties rather than the creation of new infrastructure. Whatever the cause, the data seem to demand the notch function.

To model the sharp decline at the end of World War II, we used a simple linear drop of the form

$$
\Delta_{W W 2}[P(t)]=\delta W(t),
$$

where $\delta$ is a non-negative fitting parameter and

$$
W(t)= \begin{cases}0 & , t \leq t_{1944} \\ -\left(t-t_{1944}\right) & , t_{1944}<t \leq t_{1945} \\ 1 & , t_{1945}<t .\end{cases}
$$

This drop, with no subsequent partial recovery, worked quite well in the fit. Again, this seems reasonable because the sharp decline was most likely caused by the destruction of production facilities in the closing years of the war.

Adding the terms (7.1) and (7.3) to the boxcar model (6.1) gives

$$
\begin{aligned}
P(t)= & P_{0} \exp \left\{\alpha t-\beta\left[\int_{t-\tau}^{t} b\left(t^{\prime}-t ; \tau\right) T\left(t^{\prime}\right) d t^{\prime}\right.\right. \\
& \left.\left.-\int_{-\tau}^{0} b\left(t^{\prime} ; \tau\right) T\left(t^{\prime}\right) d t^{\prime}\right]\right\} \\
& +\gamma D(t)+\delta W(t),
\end{aligned}
$$


where $b\left(t^{\prime \prime} ; \tau\right)$ is defined by Eqn. (6.2). This model has 6 free parameters: $P_{0}, \alpha, \beta, \tau, \gamma$, and $\delta$. The parameter estimates obtained by fitting it to the production data are given in Table 4 which also repeats the estimates for the model (6.1) for comparison. Note the significant changes in the parameters common to both fits. Note also that the addition of the two new terms nearly halved the SSR value while boosting the percentage of variance explained by the model from $99.64 \%$ to $99.82 \%$. We performed an F-test to be sure that this represents a significant improvement. In the full model, we were fitting 121 data points with 6 free parameters, and the null hypothesis was

$$
\mathcal{H}_{0}:\{\gamma=0, \delta=0\},
$$

so $m=121, n=6$, and $n_{h}=2$. The value of the $u$ statistic (2.9) is thus

$$
u=\left(\frac{1.321 \times 10^{6}}{6.548 \times 10^{5}}-1\right) \times \frac{115}{2}=58.5
$$

which is much larger than $F_{0.9999}(2,115)=10.01$, so the null hypothesis is rejected at the $99.99 \%$ level.

A plot of the fit is given in Figure 8. Comparing it with the one given in Figure 6 shows that it tracks the data better, not only in the years 1929-1935 and 1944-1945, but also in the years 1900-1929, 1935-1944, and 1945-1960. This improvement is also quite evident in the plot of the residuals given in Figure 9. Comparing them with the ones given in Fig. 7 shows that the new terms have not only eliminated the sharp drops, but also have eliminated the sytematic variation with temperature in the years 1910-1970. Thus the new terms free the boxcar averaging term to completely capture the temperature modulation.

Even though the new model explains $99.82 \%$ of the total variance in the data, we shall see in the following that further significant improvements can by obtained by including additional free parameters to give a more realistic estimate of the temperature averaging function. Before doing that, however, we will check whether the addition of the terms for the Depression and the War makes it possible to drop some of the parameters previously included in the model. We begin by adding the new terms to the unmodulated exponential to get the model

$$
P(t)=P_{0} \exp (\alpha t)+\gamma D(t)+\delta W(t),
$$

which gives the fit shown in Figure 10. Note that the the drop for the Great Depression is too large and the drop for World War II is almost negligible. 
The fit does not track the data, and though we do not show them here, the residuals display the inverse temperature signature.

The next step is to check whether the inverse temperature dependence can be adequately modelled by adding the new terms to the original Rust-Kirk model (2.3), i.e.,

$$
P(t)=P_{0} \exp \{\alpha t-\beta[T(t)-T(0)]\}+\gamma D(t)+\delta W(t) .
$$

Using the optimal smoothing spline representation for $T(t)$ to fit this expression gave the curve shown in Figure 11. The improvement over the fit shown in Figure 10 is quite dramatic. The SSR values for the two models are $\rho_{1}=5.842 \times 10^{6}$ for (7.7) and $\rho=1.115 \times 10^{6}$ for (7.8) so by (2.9),

$$
u=\left(\frac{5.842 \times 10^{6}}{1.115 \times 10^{6}}-1\right) \times \frac{(121-5)}{1}=492,
$$

which is much larger than $F_{0.9999}(1,116)=16.2$. Thus the significance of the parameter $\beta$ is adequately established. The important question now becomes whether the parameter $\beta$ is adequate by itself of whether we need to add the lag parameter $\tau$.

Basically, the problem is to test the augmented boxcar model (7.5) against the augmented Rust-Kirk model (7.8). Using the definition (6.2), it is not difficult to show that the latter is the limit of the former as $\tau \rightarrow 0$. But it is not simply a case of testing the fit shown in Figure 8 against the one shown in Figure 11 since the latter used the optimal spline representation of $T(t)$ while the former used the interpolating spline. To get two comparable fits, we used the optimal smoothing spline representation with the model (7.5) to get the fit shown in Figure 12 which appears to be only marginally better than the one in Figure 11. It is important, therefore, to test the null hypothesis

$$
\mathcal{H}_{0}: \quad \tau=0 \text {. }
$$

The SSR values were $\rho=1.049 \times 10^{6}$ for $(7.5)$ and $\rho_{1}=1.115 \times 10^{6}$ for $(7.8)$, so (2.9) becomes

$$
u=\left(\frac{1.115 \times 10^{6}}{1.049 \times 10^{6}}-1\right) \times \frac{(121-6)}{1}=7.24
$$

which is larger than $F_{0.99}(1,115)=6.86$. Thus the null hypothesis is rejected and the parameter $\tau$ is significant at the $99 \%$ level.

Replacing the smoothing spline representation of $T(t)$ with the interpolating spline gives a marked improvement in the boxcar fit, reducing the SSR 
value from $1.049 \times 10^{6}$ to $6.548 \times 10^{5}$, with no additional parameters. Comparing Figures 8 and 12 shows that most of this improvement comes from a better fit in the years 1970-1990. It is clear from the preceding that all of the 6 free parameters in (7.5) are necessary to obtain this improved fit.

\section{The Temperature Averaging Function}

The boxcar model assumes a constant dependence on the temperatures at every moment in a preceding time interval. Clearly, it is more realistic to assume that the level of dependence decreases with increasing time delay. Therefore we consider models of the form

$$
\begin{aligned}
P(t)= & P_{0} \exp \left\{\alpha t-\beta\left[\int_{t-\tau}^{t} w\left(t^{\prime}-t ; \tau\right) T\left(t^{\prime}\right) d t^{\prime}\right.\right. \\
& \left.\left.\quad-\int_{-\tau}^{0} w\left(t^{\prime} ; \tau\right) T\left(t^{\prime}\right) d t^{\prime}\right]\right\} \\
& +\gamma D(t)+\delta W(t),
\end{aligned}
$$

where $w\left(t^{\prime \prime} ; \tau\right)$ is a tapered temperature averaging function, dependent on a free parameter $\tau$, and constrained only by the properties:

$$
\left.\begin{array}{l}
w\left(t^{\prime \prime} ; \tau\right)>0,-\tau<t^{\prime \prime} \leq 0 \\
w\left(t^{\prime \prime} ; \tau\right)=0, \text { otherwise }
\end{array}\right\}
$$

and

$$
\int_{-\tau}^{0} w\left(t^{\prime \prime} ; \tau\right) d t^{\prime \prime}=1
$$

The relationship between the temperature time series and the unknown averaging function is shown schematically in Fig. 13.

One strategy for determining $w\left(t^{\prime \prime} ; \tau\right)$ is to assume a functional form for it, substitute this function into (8.1), and fit the resulting expression to the data. The assumed function will have a free parameter $\tau$ characterizing the length of the memory. The boxcar averaging function (6.2) is the simplest example of such a functional form.

Another simple example is the straight-line, triangular function defined by

$$
w\left(t^{\prime \prime} ; \tau\right)= \begin{cases}\frac{2}{\tau}+\frac{2}{\tau^{2}} t^{\prime \prime} & , \quad-\tau \leq t^{\prime \prime} \leq 0 \\ 0 & , \text { otherwise }\end{cases}
$$

This function is completely specified by the parameter $\tau$ and satisfies the unit area constraint (8.3), but fitting it to the production data gives approximately the same SSR as the boxcar. To make significant improvements in the fit, it is necessary to introduce additional free parameters defining the shape of the averaging function. 


\section{A Quadrilateral Averaging Function}

Consider the quadrilateral function shown schematically in Figure 14. The sides of the quadrilateral are defined by the coordinate axes and the two solid lines intersecting at the point $\left(-\tau_{1}, h_{1}\right)$. If the parameters $\tau, \tau_{1}, h_{1}$ and $h_{0}$ are chosen to give the quadrilateral unit area, then

$$
h_{0}=\frac{1}{\tau_{1}}\left(2-\tau h_{1}\right),
$$

and the two line segments

$$
\begin{array}{ll}
w_{1}\left(t^{\prime \prime} ; \tau, \tau_{1}, h_{1}\right)=\frac{\tau h_{1}}{\tau-\tau_{1}}+\frac{h_{1}}{\tau-\tau_{1}} t^{\prime \prime} & ,-\tau \leq t^{\prime \prime} \leq-\tau_{1}, \\
w_{2}\left(t^{\prime \prime} ; \tau, \tau_{1}, h_{1}\right)=\frac{2-\tau h_{1}}{\tau_{1}}+\frac{2-\left(\tau+\tau_{1}\right) h_{1}}{\tau_{1}^{2}} t^{\prime \prime}, & -\tau_{1} \leq t^{\prime \prime} \leq 0 .
\end{array}
$$

define a piecewise linear function

$$
w\left(t^{\prime \prime} ; \tau, \tau_{1}, h_{1}\right)= \begin{cases}w_{1}\left(t^{\prime \prime} ; \tau, \tau_{1}, h_{1}\right) & ,-\tau \leq t^{\prime \prime} \leq-\tau_{1} \\ w_{2}\left(t^{\prime \prime} ; \tau, \tau_{1}, h_{1}\right) & ,-\tau_{1} \leq t^{\prime \prime} \leq 0 \\ 0 & , \text { otherwise }\end{cases}
$$

which satisfies (8.2) and (8.3). Using this averaging function in the general model (8.1) gives

$$
\begin{aligned}
P(t)= & P_{0} \exp \left\{\alpha t-\beta\left[\int_{t-\tau}^{t} w\left(t^{\prime}-t ; \tau, \tau_{1}, h_{1}\right) T\left(t^{\prime}\right) d t^{\prime}\right.\right. \\
& \left.\left.\quad-\int_{-\tau}^{0} w\left(t^{\prime} ; \tau, \tau_{1}, h_{1}\right) T\left(t^{\prime}\right) d t^{\prime}\right]\right\} \\
& +\gamma D(t)+\delta W(t) .
\end{aligned}
$$

If $T(t)$ is the interpolating spline representation of the temperatures, then for any values of the parameters $\tau, \tau_{1}$, and $h_{1}$, the integrals

$$
\begin{aligned}
\int_{t-\tau}^{t} w\left(t^{\prime}-t ; \tau, \tau_{1}, h_{1}\right) T\left(t^{\prime}\right) d t^{\prime} & =\int_{t-\tau}^{t-\tau_{1}} w_{1}\left(t^{\prime}-t ; \tau, \tau_{1}, h_{1}\right) T\left(t^{\prime}\right) d t^{\prime} \\
& +\int_{t-\tau_{1}}^{t} w_{2}\left(t^{\prime}-t ; \tau, \tau_{1}, h_{1}\right) T\left(t^{\prime}\right) d t^{\prime} \\
\int_{-\tau}^{0} w\left(t^{\prime} ; \tau, \tau_{1}, h_{1}\right) T\left(t^{\prime}\right) d t^{\prime} & =\int_{-\tau}^{-\tau_{1}} w_{1}\left(t^{\prime} ; \tau, \tau_{1}, h_{1}\right) T\left(t^{\prime}\right) d t^{\prime} \\
& +\int_{-\tau_{1}}^{0} w_{2}\left(t^{\prime} ; \tau, \tau_{1}, h_{1}\right) T\left(t^{\prime}\right) d t^{\prime}
\end{aligned}
$$


can be evaluated numerically, so it is possible, though difficult, to fit (9.4) to the data. The result is shown if Figure 15, and the corresponding parameter estimates are given in Table 4 . Comparing these estimates with the corresponding values for the boxcar fit shows that only $\tau$ changed significantly. Adding the two additional free parameters reduced the SSR from $\rho_{1}=6.548 \times 10^{5}$ to $\rho=5.637 \times 10^{5}$. To verify that this is a statistically significant reduction, consider the null hypothesis

$$
\mathcal{H}_{0}:\left\{\tau_{1}=\tau, h_{1}=\frac{1}{\tau}\right\},
$$

which reduces (9.4) to (7.5). The $u$-statistic (2.9) becomes

$$
u=\left(\frac{6.548 \times 10^{5}}{5.637 \times 10^{5}}-1\right) \times \frac{113}{2}=9.131,
$$

and $F_{0.9995}(2,113)=8.139$, so the null hypothesis is rejected at the $99.95 \%$ level of significance.

Using the estimates of $\tau, \tau_{1}$, and $h_{1}$ in (9.1) gives $h_{0}=0.084$, which completes the specification of the shape and dimensions of the quadrilateral window. A plot is given in Figure 16 which also shows the boxcar window for comparison. The quadrilateral window is surprisingly flat in the interval $[-5.1,0]$.

\section{A Transfer Function for the Modulation}

Changing from a boxcar to a quadrilateral averaging function required the addition of two new parameters defining the intersection $\left(\tau_{1}, h_{1}\right)$ of the two piecewise linear segments. A logical next step would be to introduce another node $\left(-\tau_{2}, h_{2}\right)$ defined by two more free parameters. The new approximation would be accepted only if the new model gave a statistically significant reduction in the SSR. If so, the whole procedure could be repeated and continued so long as each new pair of free parameters produced a significant improvement in the fit. But implementing this strategy would be clumsy because the piecewise approximations are cumbersome, so we instead sought a discrete estimate of the averaging function by replacing the integrals in (8.1) with approximating quadrature sums.

To simplify the development, we temporarily drop the terms for the Depression and the War and work with the reduced model

$$
P(t)=P_{0} \exp \left\{\alpha t-\beta\left[\int_{t-\tau}^{t} w\left(t^{\prime}-t ; \tau\right) T\left(t^{\prime}\right) d t^{\prime}-\int_{-\tau}^{0} w\left(t^{\prime} ; \tau\right) T\left(t^{\prime}\right) d t^{\prime}\right]\right\} .
$$


Since both temperature and fuel production are tabulated at yearly intervals, we used a quadrature mesh with $\Delta t^{\prime}=1$ to approximate the integrals, i.e.,

$$
\begin{gathered}
\int_{t-\tau}^{t} w\left(t^{\prime}-t ; \tau\right) T\left(t^{\prime}\right) d t^{\prime} \cong \sum_{j=-\tau}^{0} Q_{j} w(j ; \tau) T(t+j), \\
\int_{-\tau}^{0} w\left(t^{\prime} ; \tau\right) T\left(t^{\prime}\right) d t^{\prime} \cong \sum_{j=-\tau}^{0} Q_{j} w(j ; \tau) T(j),
\end{gathered}
$$

where the $Q_{j}$ are the quadrature coefficients, and $\tau$ is assumed to have an integer value. Since the integrands depend on measured data, we used the trapezoidal rule for the quadrature, so

$$
\begin{aligned}
& \left\{Q_{-\tau}, Q_{-\tau+1}, Q_{-\tau+2}, \ldots, Q_{-2}, Q_{-1}, Q_{0}\right\}=\left\{\frac{\Delta t^{\prime}}{2}(1,2,2, \ldots, 2,2,1)\right\} \\
& =\left\{\frac{1}{2}, 1,1, \ldots, 1,1, \frac{1}{2}\right\} \text {. }
\end{aligned}
$$

Substituting (10.2) and (10.3) into (10.1) and simplifying gives

$$
P(t)=P_{0} \exp \left\{\alpha t-\beta \sum_{j=-\tau}^{0} Q_{j} w(j ; \tau)[T(t+j)-T(j)]\right\},
$$

which, taking $i=-j$ and reversing the order of the summation, becomes

$$
P(t)=P_{0} \exp \left\{\alpha t-\beta \sum_{i=0}^{\tau} Q_{-i} w(-i ; \tau)[T(t-i)-T(-i)]\right\} .
$$

Defining a new set of coefficients:

$$
\beta_{i} \equiv \beta Q_{-i} w(-i ; \tau), \quad i=0,1,2, \ldots, \tau,
$$

gives

$$
P(t)=P_{0} \exp \left\{\alpha t-\sum_{i=0}^{\tau} \beta_{i}[T(t-i)-T(-i)]\right\} .
$$

Equation (8.2) requires

$$
w(-\tau ; \tau)=0, \quad \beta_{\tau}=0,
$$

so, defining a new integer

$$
N \equiv \tau-1
$$

gives

$$
P(t)=P_{0} \exp \left\{\alpha t-\sum_{i=0}^{N} \beta_{i}[T(t-i)-T(-i)]\right\}
$$


which is a natural generalization of the original Rust-Kirk model. In fact, if $N=0$, the above model reduces exactly to (2.3) with $\beta=\beta_{0}$.

Restoring the terms for the Great Depression and World War II gives

$$
P(t)=P_{0} \exp \left\{\alpha t-\sum_{i=0}^{N} \beta_{i}[T(t-i)-T(-i)]\right\}+\gamma D(t)+\delta W(t),
$$

The estimation procedure was to fix the value of $N$ and fit this expression to the data, in the process computing estimates for $P_{0}, \alpha, \beta_{0}, \beta_{1}, \ldots, \beta_{N}, \gamma$, and $\delta$. The $\beta_{i}$ values can then be used with the constraint (8.3) and Eq. (10.7) to backsolve for $\beta$ and the required $w(-i ; \tau)$ values. Using trapezoidal quadrature to evaluate the integral in (8.3) gives

$$
\sum_{j=0}^{N} Q_{-j} w(-j ; \tau) \cong 1
$$

Assuming exact equality, multiplying by $\beta$ and using (10.7) gives

$$
\beta=\sum_{j=0}^{N} \beta_{j} .
$$

Substituting this result into (10.7) and back-solving gives

$$
w(-i ; \tau)=\frac{\beta_{i}}{Q_{-i} \sum_{j=0}^{n} \beta_{j}}, \quad i=0,1,2, \ldots, N,
$$

where the $Q_{-i}$ are given by (10.4). Combining these results with Eq. (10.9) gives a collection of points

$$
\mathcal{M}=\{[-i, w(-i ; \tau)], \quad i=0,1,2, \ldots, N, \tau\}
$$

which constitutes a discrete transfer function approximation to the temperature averaging function.

Our strategy for choosing the value of $\tau$ was to fit (10.12) to the data with successive values of $N$, starting with $N=1$ and continuing for as long as each new fit gave a statistically significant improvement in the SSR at the $95 \%$ level. The estimated parameters for $N=6,7,8,9$, and 10 are given in Table 5. The table gives only the sum of the $\beta_{i}$, but the estimates of the individual ordinates $w(-i ; N+1)$ are plotted in Figure 17, with the $\pm 1 \sigma$ uncertainties plotted for the case $N=8$. None of the estimates in the table vary widely from fit to fit and all are roughly comparable to those in Table 4 for the boxcar and quadrilateral estimates. In particular, the value for $\alpha$ is very stable. In 
Fig. 17 the individual ordinates do not change drastically from one fit to the next, and the estimates of the $w(-i ; N+1)$ are all positive for $N \leq 8$, but for $N \geq 9$, some of them are negative. Thus, $N=8$ is the largest value for which the estimates satisfy the constraints (8.2).

For each new value of $N$ we performed an F-test on the null hypothesis

$$
\mathcal{H}_{0}: \beta_{N}=0
$$

to determine whether the new parameter produced a statistically significant reduction in the SSR. The values of the $u$-statistic are tabulated in Table 5 , together with the $95 \%$-point for the approptiate $F$-distribution, i.e., $F_{0.95}(1,121-$ $n$ ) where $n$ is the number of free parameters in the model and 121 is the number of data points. Comparing the numbers in the two rows reveals that the null hypothesis should be rejected for all values up to and including $N=8$ and accepted for $N \geq 9$. There is a satisfying consistency in the fact that the threshold for acceptance is so sharp and corresponds exactly to the onset of violations of the nonnegativity constraints (8.2). Also, at $N=8$, the value of $\beta$ is maximized and the values of $\gamma$ and $\delta$ are minimized. This means that the percentage of the total variation explained by the temperature modulation is maximized while the variation assigned to the Great Depression and World War II is minimized. Accordingly we adopted the value $N=8$, i.e.

$$
\tau=9, \quad \beta_{9}=0, \quad w(-9 ; 9)=0,
$$

and used the corresponding values of $\beta_{0}, \beta_{1}, \ldots, \beta_{8}$ to compute the desired estimates of $w(0 ; 9), w(-1 ; 9), \ldots, w(-8 ; 9)$. The results are plotted in Figure 18 as small circles with the $\pm 1 \sigma$ error bars attached. The figure also shows the boxcar and quadrilateral estimates for comparison and a smoothing spline fit that will be discussed in the next sectiom.

The ten circles plotted in Figure 18 define a transfer function whose shape is similar to that of the quadrilateral estimate. Only two of the ten $\pm 1 \sigma$ intervals fail to include the quadrilateral averaging function. Both functions exhibit a flat plateau behavior between $\tau=-4.5$ and $\tau=-1.5$. This indicates a long-lived temperature influence which, though surprising, is demanded by the data. A possible cause might be the time required to increase or decrease production capacity. Opening new mines or oilfields or building new refineries are not done instantaneously, and once in place, there is undoubtedly a great reluctance to shut them down until they become completely unprofitable.

The fit to the production data is plotted in Figure 19. It is not surprising that it is very similar to the one shown in Figure 15 for the quadrilateral. The latter used 8 free parameters to give SSR $=5.637 \times 10^{5}$ while the transfer model 
employed 13 free parameters to obtain SSR $=5.341 \times 10^{5}$. Since the former was not obtained from the latter by simply adding 5 more parameters, it is not possible to directly compare them via the F-test. Nevertheless, we have shown that all of the parameters in the transfer function model are significant, so the transfer function is a better estimate of the true averaging function.

\section{Uniqueness of the Modulation}

Although the production data support the 13 free parameters in the transfer function model, it is natural to wonder about the uniqueness of the temperature record as a modulating function. Given so many free parameters, would it be possible to obtain as good a fit using some completely different time-series for the modulating function? To test this possibility, we generated 10,000 random time-series with the same number of points, the same mean, and the same variance about that mean as the measured temperature record. More precisely, we generated 10,000 time-series of the form

$$
T\left(y_{i}\right)=T_{a v}+\eta\left(y_{i}\right), \quad y_{i}=1851,1852, \ldots, 1991,
$$

where $T_{a v}$ is the average of the 141 temperatures in the measured time-series, and the $\eta\left(y_{i}\right)$ are random samples from a normal distribution,

$$
\eta \sim n\left(0, \sigma^{2}(T)\right),
$$

where $\sigma^{2}(T)$ is the variance of the measured temperature about $T_{a v}$. We used each of these random time-series to fit the $N=8$ transfer function model to the production data and recorded the resulting SSR. The distribution of SSR values is shown in Figure 20. The mean and standard deviation were $\mathrm{SSR}_{a v}=$ $(4.2 \pm 1.1) \times 10^{6}$, and the smallest was $\mathrm{SSR}_{\min }=7.708 \times 10^{5}$ which is larger than the value $S S R=5.341 \times 10^{5}$ obtained with the real temperatures. Thus, even with 13 free parameters, the probability that a random time series will give a fit as good as the temperature record is less than 0.0001 . The production data demand the temperature time-series as the modulating function.

\section{A Spline Modulating Function}

The transfer function model included 13 free parameters, but 9 of them were required to specify the transfer function. To reduce the number of parameters, we used the CUBGCV algorithm (Hutchinson, 1986) to fit an optimal 
smoothing spline to the 9 discrete transfer ordinates. This gave a continuous averaging function which is shown as the solid curve in Figure 18. Note that the time span of the function is 9.1370 years because we did not require the spline to satisfy the restriction $w(-9 ; 9)=0$. In fitting the spline, we weighted the discrete ordinates inversely with their $\pm 1 \sigma$ uncertainties, except in the case of $w(-9 ; 9)$ which had been assigned the value zero. To that point, we assigned an uncertainty equal to the largest of the uncertainties for the other ordinates. The smoothing constant $s$ was chosen by the CUBGCV subroutine to minimize the cross-validation statistic, i.e., to minimize the expected mean square error at the data points. The value $s=0$ would have given an interpolating spline incorporating the point-to-point variations due to the statistical uncertainties. A very large value $(s \rightarrow \infty)$ would have given a least squares straight line, which would have been far too smooth. The zero point $t=-9.1370$ was determined by a variant of the ZEROIN algorithm (Dekker, 1969) coded at the Sandia Laboratories (Shampine and Watts, 1970). Numerically integrating the spline from $t=-9.1370$ to $t=0$ gave the value 1.00221 , so the spline function was multiplied by the reciprocal of that value to force it to satisfy the constraint (8.3). It is this renormalized function that is plotted in Figure 18.

If $w_{s}(t)$ denotes the spline averaging function, then the corresponding lagged response model can be written [cf. Eqn. (8.1)]

$$
\begin{aligned}
P(t)= & P_{0} \exp \left\{\alpha t-\beta\left[\int_{t-\tau_{0}}^{t} w_{s}\left(t^{\prime}-t\right) T\left(t^{\prime}\right) d t^{\prime}\right.\right. \\
& \left.\left.\quad-\int_{-\tau_{0}}^{0} w_{s}\left(t^{\prime}\right) T\left(t^{\prime}\right) d t^{\prime}\right]\right\} \\
& +\gamma D(t)+\delta W(t),
\end{aligned}
$$

where $\tau_{0}=9.1370$. This model has 5 free parameters, $P_{0}, \alpha, \beta, \gamma$, and $\delta$. Using the interpolating spline representation for $T(t)$ and fitting (12.1) gives the solid curve in Figure 21 and the parameter estimates in the last column of Table 4. These estimates do not differ significantly from the corresponding values for the quadrilateral averaging function. The SSR values for the two models agree to four significant digits even though the latter has 3 additional adjustable parameters. This is not surprising since the two averaging functions (shown in Figure 18) are so similar everywhere except the subinterval $t=-1$ to $t=0$.

To isolate the effect of the temperature modulation, we also fit the data with a model of the form

$$
P(t)=P_{0} \exp \left(\alpha_{1} t\right)+\gamma_{1} D(t)+\delta_{1} W(t),
$$


where $\alpha_{1}, \gamma_{1}$, and $\delta_{1}$ were fixed at the values obtained from the spline averaging model (the values in the last column of Table 4), but the parameter $P_{0}$ was allowed to vary because holding it similarly fixed would be tantamount to the unjustified assumption that the modulation was exactly zero at epoch 1870.0. The fit gave the dashed curve shown in Figure 21. Since the exponential rate and the drops for the Great Depression and World War II were identical to those in the spline model, the difference between the two curves measures the magnitude of the temperature modulation. The residuals for the two models are plotted in Figure 22 where again, the difference between the two curves measures the modulation. This modulation is rendered even more explicitly in Figure 23 where the solid curve is a plot of the difference between the two models in Figure 21. The perturbations for the Depression and the War are also plotted as dashed curves for comparison. It is important to remember that these perturbations are additive effects, independent of the temperature modulation.

It is apparent from Figure 23 that the underlying exponential growth produces a corresponding inflation of the modulation which makes it difficult to compare the values at different times during the record. In Figure 24 we have replotted the three curves as fractions of the predicted total production in the same year. More precisely, for each year we divided the deviations plotted in Figure 23 by the predicted total production for that year, i.e., by the value plotted on the solid curve in Figure 21. Comparing the solid curve with the smoothing spline fit to the temperatures, given in Figure 3, shows that the period of generally increasing temperatures from 1887 to 1940 produced a decrease in the modulation from $+33 \%$ of the total in 1888 to $-9 \%$ of the total in 1942. This decline, though gradual, was more than three times greater than the $12 \%$ net decrease caused by the Great Depression and more than twice the $17 \%$ drop caused by the destruction of World War II. The magnitudes of these perturbations were also smaller than the increase in the modulation from $-9 \%$ of the total in 1942 to $+14 \%$ of the total in 1972 which was produced by the falling temperatures between 1940 and 1968. Finally, the increasing temperatures from 1969 to 1990 produced a drop in the modulation from $14 \%$ of the total in 1972 to $-19 \%$ of the total in 1990. This drop was greater than the combined total for the depression and the war.

\section{The Gaia Hypothesis}

When they wrote Paper I, Rust and Kirk were not aware of the Gaia hypothesis which had been posed almost a decade earlier [Lovelock (1972), Lovelock 
and Margulis (1974), Margulis and Lovelock (1974)]. This hypothesis regards the atmosphere "... as a component part of the biosphere rather than as a mere environment for life," and suggests that "... the total ensemble of living organisms which constitute the biosphere can act as a single entity to regulate chemical composition, surface $\mathrm{pH}$ and possibly also climate." In particular, Lovelock and Margulis point out that throughout the approximately $3 \times 10^{9}$ year history of life on Earth, the temperature of the Earth's surface has remained remarkably constant in spite of the fact that the luminosity of the Sun has increased by approximately tenfold during the same period. One important factor in maintaining this temperature constancy is the presence of greenhouse gases in the atmosphere, and the most effective of these interact strongly with the biosphere. According to the Gaia hypothesis, the biosphere adjusts the concentrations of these gases as one of the controls for maintaining the optimal surface temperature for life.

Carbon dioxide is one of the important greenhouse gases. Its atmospheric concentration is currently increasing at an exponential rate almost identical to that for fossil fuel production (Rust et al, 1979), and approximately $58 \%$ of the fossil fuel carbon finds permanent residence in the atmosphere (Kirk and Rust, 1983). Recent updates of the measurements of Keeling and his colleagues [Boden (1988)], reveal that this rate was sufficient to raise the $\mathrm{CO}_{2}$ level by almost $11 \%$ in the years 1958 through 1987 [see also Keeling et al (1982)]. This dramatic increase is causing concern about the onset of a global greenhouse warming. If the Gaia hypothesis is correct, then the biosphere should sense such a warming and produce a feedback to counter it. Although we cannot identify a mechanism for the feedback, we note that the modulation that we have described is this paper operates in a manner consistent with the Gaia prediction.

The rapid technological progress in this century has led to the wide-spread belief that man has finally conquered Nature and is now able to control her for his own benefit. But the relentless, basically exponential growth in fossil fuel production over the last 130 years is disturbingly reminiscent of the behavior of a colony of microbes in a petri dish. There is now little doubt that such behavior will have drastic and probably negative consequences. The real debate is currently centered on the time-scale for these changes, usually with an underlying assumption that when the effects become troublesome, ameliorating technologies will be developed. But the temperature modulation of the fossil fuel production suggests that Nature may not be content to await these new technologies. In the last section we showed that the production changes associated with the temperature variations are considerably larger than those caused by the severest economic catastrophes concocted by man. There may 
be controversy about whether or not the strong warming trend of the last two decades represents the onset of the greenhouse warming, but whatever its cause, it provoked a significant, perhaps Gaiaen, response in the fossil fuel production rate. It would seem then that the recent announcement of The End of Nature (McKibben,1989) may have been premature.

We urge readers who are not already familiar with the Gaia hypothesis to consult Lovelock's very accessible books on the subject [(Lovelock, 1979, 1988, 1991)].

\section{Extrapolations}

We have demonstrated a significant inverse correlation between Northern Hemispheric land surface temperatures and the global production of fossil fuels which, in turn, is an indicator of global economic activity. The optimal smoothing spline in Figure 3 shows that the last two decades have seen the most rapid warming since the beginning of the temperature record. At the same time the modulation plots given in Figures 23 and 24 show a corresponding decline in the rate of increase in fossil fuel production. In fact there were actual declines in the total production in the years 1980-1983. It is therefore reasonable to wonder whether the current global recession may be due more to the recent temperature increase than to the success or failure of any particular economic or political system.

It is also natural to wonder about future economic activity, and in particular about what the model (12.1) predicts about future fossil fuel production. Of course, the model can make such a prediction only if it is supplied with the future temperature record which is, of course, completely unknown. But we can envisage possible future temperature scenarios and calculate the resulting predictions of fuel production. Three such scenarios are given in Figure 25 which shows three possible 20-year extensions of the optimal smoothing spline fit to the temperature record. The upper extension was obtained by linearly extrapolating the spline fit, and the lower extension was obtained by reversing that extrapolation, i.e., by reflecting it through the horizontal direction. The middle extension was obtained by simply repeating the final value of the spline for each of the 20 years. Of course the real temperature record will have large year-to-year variations, but the temperature averaging will tend to smooth out their effects on the yearly production. Therefore we ignore them and consider only the smooth extensions shown in the figure. The upper and lower scenarios hopefully represent extremes of conceivable future temperature change. The record to date does not contain a cooling period comparable to the lower sce- 
nario, and the rate of the recent warming, which the upper scenario extends, hopefully represents an upper extreme for future temperature changes.

To compute the fuel production predictions corresponding the these three scenarios, we used the model (12.1) with the parameter values from the last column of Table 4 , i.e.,

$$
\begin{aligned}
P(t)= & (183) \exp \left\{(0.0326) t-(0.77)\left[\int_{t-9.137}^{t} w_{s}\left(t^{\prime}-t\right) T\left(t^{\prime}\right) d t^{\prime}\right.\right. \\
& \left.\left.-\int_{-9.137}^{0} w_{s}\left(t^{\prime}\right) T\left(t^{\prime}\right) d t^{\prime}\right]\right\} \\
& +(93) D(t)+(213) W(t),
\end{aligned}
$$

where $D(t)$ and $W(t)$ are given by Equations (7.2) and (7.4) respectively, and $w_{s}(t)$ is the averaging function shown as a smooth curve in Figure 18. For $T(t)$, we used interpolating spline fits to the three extended temperature records. For the years with actual temperature measurements, these fits were identical to the interpolating spline shown in Figure 3. The integrals were computed numerically using the Gauss-Kronrod automatic integrator QAG (Piessens et al, 1983). The results are plotted in Figure 26 where the data points and the solid curve passing through them are the same as those in Figure 21, and each of the extrapolations corresponds to the temperature extrapolation with the same line pattern in Figure 25.

For all of the temperature extrapolations, the model initially predicts a few years with no increases, or even decreases, in fuel production while the lagged averaging function works through the recent temperature increases. After the initial hiatus, the lower temperature extrapolation (long dashes) predicts a spectacular increase in fuel production with almost a $200 \%$ increase by the end of the twenty years. Of course this assumes the existence of sufficient readily available fuel reserves and no large scale changes to other energy sources during the period. The constant temperature extrapolation (short dashes) predicts an eventually constant rate exponential growth which would produce an increase of about $70 \%$ in the total production by the end of the period. Although this prediction appears to be more consistent with the historical record than the other two, it depends on a temperature scenario that, with our present state of knowledge, is just as improbable as the other two. The higher temperature scenario predicts a virtual halt to all increases in fossil fuel production. Should the temperature scenario upon which it depends actually materialize, there could be little remaining doubt that the predicted greenhouse warming had arrived. While the concomitant reduction in fossil fuel production might have a desirable ameliorating effect, the associated economic adjustments might be severe, especially if global population growth continues at the present rate. 


\section{Acknowledgements}

We would like to thank James L. Blue and Robert E. Chapman for their very helpful criticism and advice, Philip D. Jones and Gregg Marland for providing us with unpublished data, Julia A. Watts, Thomas A. Boden and the Carbon Dioxide Information Analysis Center for providing computer readable records of published data, and Ronald F. Boisvert for expert advice on numerical analysis and programming considerations.

\section{References}

[1] Boden, T.A. (1988) Numeric data package NDP001.REV, ORNL/CDIC17, Carbon Dioxide Information Analysis Center, Oak Ridge, TN.

[2] Boisvert, R.F., Howe, S.E. and Kahaner, D.K (1984) The guide to available mathematical software (GAMS), PB 84-171305, National Technical Information Service, Springfield, VA.

[3] Brinkmann, W.A.R. (1976) Surface temperature trend for the Northern Hemisphere - updated, Quaternary Research 6, pp 355-358.

[4] Craven, P. and Wahba, G. (1979) Smoothing noisy data with spline functions, Numerische Mathematik 31 pp. 377-403.

[5] Dekker, T.J. (1969) Finding a zero by means of successive linear interpolation, in Constructive Aspects of the Fundamental Theorem of Algebra, B. Dejon and P. Henrici, eds., Wiley-Interscience, New York.

[6] Golub, G.H. and Pereyra, V. (1973) The differentiation of pseudo-inverses and nonlinear least squares problmes whose variables separate, SIAM Jour. of Numerical Analysis 10 pp. 413-432.

[7] Hutchinson, M.F. (1986) Algorithm 642: A fast procedure for calculating minimum cross-validation cubic smoothing splines, $A C M$ Transactions on Mathematical Software 12, pp. 150-153.

[8] Jones, P.D., Raper, S.C.B., Bradley, R.S., Diaz, H.F., Kelly, P.M., and Wigley, T.M.L. (1986) Northern Hemisphere surface air temperature variations: 1851-1984, Jour. of Climate and Appl. Meteor. 25, pp.161-179.

[9] Jones, P.D. (1988) Hemispheric surface air temperature variations: recent trends and an update to 1987, Jour. of Climate 1, pp.654-659. 
[10] Jones, P.D. (1992) Private communication.

[11] Kahaner, D.K., Moler, C.B. and Nash, S.G. (1989) Numerical Methods and Software, Prentice Hall, Englewood Cliffs, NJ.

[12] Keeling, C.D., Bacastow, R.B. and Whorf, T.P. (1982) Measurements of the concentration of carbon dioxide at Mauna Loa Observatory, Hawaii, in Carbon Dioxide Review: 1982, W.C. Clark, ed., pp. 375-385, Oxford University Press, New York.

[13] Kirk, B.L. and Rust, B.W. (1983) The solar cycle effect on atmospheric carbon dioxide levels, in Weather and Climate Responses to Solar Variations, B.M. McCormac, ed., pp 129-136, Colorado Associated University Press, Boulder, CO.

[14] Lovelock, J.E. (1972) Gaia as seen through the atmosphere, Atmospheric Enviroment 6, pp. 579-580.

[15] Lovelock, J.E. (1979) Gaia: A New Look at Life on Earth, Oxford University Press, Oxford.

[16] Lovelock, J.E. (1988) The Ages of Gaia: A Biography of Our Living Earth, W.W. Norton \& Co., New York.

[17] Lovelock, J.E. (1991) Healing Gaia: Practical Medicine for the Planet, Harmony Books, New York.

[18] Lovelock, J.E. and Margulis, L. (1974) Atmospheric homeostatis by and for the biosphere: the gaia hypothesis, Tellus XXVI, pp. 2-10.

[19] McKibben, W. (1989) The End of Nature, Random House, New York.

[20] Margulis, L. and Lovelock, J.E. (1974) Biological modulation of the Earth's atmosphere, Icarus 21, pp. 471-489.

[21] Marland, G. (1989) Fossil fuels $\mathrm{CO}_{2}$ emissions: three countries account for $50 \%$ in 1986, CDIAC Communications, Oak Ridge National Laboratory (Winter, 1989) pp. 1-3.

[22] Marland, G. (1992) Private communication.

[23] Marland, G. and Boden, T. (1992) $\mathrm{CO}_{2}$ Emissions - Modern Record, in TRENDS '91: A Compendium of Data on Global Change Highlights, T.A. Bodeb, R.J. Sepanski, and F.W. Stoss, eds., pp 41-47, ORNL/CDIAC-49, Oak Ridge National Laboratory, Oak Ridge, Tennessee. 
[24] Mitchell, J.M., Jr. (1961) Recent secular changes of global temperature, Ann. New York Acad. Sci. 95, pp.235-250.

[25] Piessens, R., de Donker, E. and Kahaner, D. (1983) QUADPACK: A Subroutine Package for Automatic Integration, Springer-Verlag, New York.

[26] Rust, B.W., Rotty, R.M. and Marland, G. (1979) Inferences drawn from atmospheric $\mathrm{CO}_{2}$ data, Jour. of Geophys. Res. 84, No. C6, pp. 3115-3122.

[27] Rust, B.W. and Kirk, B.L. (1982) Modulation of fossil fuel production by global temperature variations, Enviroment International 7, pp. 419-422.

[28] Shampine, H.F. and Watts, H.A. (1970) ZEROIN, A Root-Solving Code, SC-TM-70-631, Sandia Laboratories, Albuquerque, NM.

[29] Wolfe, C.M., Rust, B.W., Dunn, J.H. and Brown, I.E. (1987) An Interactive Nonlinear Least Squares Program, NBS Tech. Note 1238, National Bureau of Standards, Gaithersburg, MD. 


\section{Tables}

Table 1: Parameter estimates from fits to the old fossil fuel production data (1870-1974) using the Mitchell-Brinkmann temperature record.

\begin{tabular}{||l|ccc|cc||}
\hline \hline Model & $\begin{array}{c}P_{0} \\
{\left[10^{6} \text { tons }\right]}\end{array}$ & $\begin{array}{c}\alpha \\
{\left[\mathrm{yr}^{-1}\right]}\end{array}$ & $\begin{array}{c}\beta \\
{\left[\left({ }^{\circ} \mathrm{C}\right)^{-1}\right]}\end{array}$ & $\begin{array}{c}\text { SSR } \\
{\left[10^{6} \text { tons }\right]^{2}}\end{array}$ & $R^{2}$ \\
\hline Exponential & $122 \pm 9$ & $.0345 \pm .0008$ & & $4.454 \times 10^{6}$ & .9678 \\
Rust-Kirk & $176 \pm 5$ & $.0327 \pm .0003$ & $1.24 \pm .06$ & $7.822 \times 10^{5}$ & .9943 \\
\hline \hline
\end{tabular}

Table 2: Parameter estimates from fits to the updated fossil fuel production data (1870-1990) using the Jones temperature record.

\begin{tabular}{||l|cccc|cc||}
\hline \hline Model & $\begin{array}{c}P_{0} \\
{\left[10^{6} \text { tons }\right]}\end{array}$ & $\begin{array}{c}\alpha \\
{\left[\mathrm{yr}^{-1}\right]}\end{array}$ & $\begin{array}{c}\beta \\
\left.\left[{ }^{\circ} \mathrm{C}\right)^{-1}\right]\end{array}$ & $\begin{array}{c}\tau \\
{[\mathrm{yr}]}\end{array}$ & $\begin{array}{c}\text { SSR } \\
{\left[10^{6} \text { tons }\right]^{2}}\end{array}$ & $R^{2}$ \\
\hline Exponential & $174 \pm 10$ & $.0302 \pm .0006$ & & & $7.324 \times 10^{6}$ & .9798 \\
Rust-Kirk & $139 \pm 6$ & $.0346 \pm .0005$ & $.58 \pm .05$ & & $3.227 \times 10^{6}$ & .9911 \\
Lagged R-K & $172 \pm 6$ & $.0333 \pm .0004$ & $.97 \pm .06$ & $5.6 \pm .4$ & $1.651 \times 10^{6}$ & .9955 \\
\hline \hline
\end{tabular}

Table 3: Parameter estimates from fits using boxcar averaging on spline representations of the Jones temperature record.

\begin{tabular}{||c|cccc|cc||}
\hline \hline$T(t)$ & $\begin{array}{c}P_{0} \\
{\left[10^{6} \text { tons }\right]}\end{array}$ & $\begin{array}{c}\alpha \\
{\left[\mathrm{yr}^{-1}\right]}\end{array}$ & $\begin{array}{c}\beta \\
\left.\left[{ }^{\circ} \mathrm{C}\right)^{-1}\right]\end{array}$ & $\begin{array}{c}\tau \\
{[\mathrm{yr}]}\end{array}$ & $\begin{array}{c}\text { SSR } \\
{\left[10^{6} \text { tons }\right]^{2}}\end{array}$ & $R^{2}$ \\
\hline Sm. Spline & $170 \pm 6$ & $.0336 \pm .0003$ & $1.04 \pm .07$ & $12.0 \pm .9$ & $1.691 \times 10^{6}$ & .9953 \\
Int. Spline & $160 \pm 4$ & $.0336 \pm .0003$ & $0.86 \pm .04$ & $8.4 \pm .3$ & $1.321 \times 10^{6}$ & .9964 \\
\hline \hline
\end{tabular}


Table 4: Parameter estimates from fits using various estimates of the averaging on the interpolating spline representation of the Jones temperature record.

\begin{tabular}{|c|c|c|c|c|}
\hline Model & Boxcar (6.1) & Boxcar (7.5) & Quadrilateral & Spline (12.1) \\
\hline$P_{0} \quad\left[10^{6}\right.$ tons $]$ & $160 \pm 4$ & $186 \pm 5$ & $184 \pm 4$ & $183 \pm 4$ \\
\hline$\alpha \quad\left[\mathrm{yr}^{-1}\right]$ & $.0336 \pm .0003$ & $.0325 \pm .0002$ & $.0326 \pm .0002$ & $.0326 \pm .0002$ \\
\hline$\beta \quad\left[\left({ }^{\circ} C\right)^{-1}\right]$ & $.86 \pm .04$ & $.77 \pm .03$ & $.77 \pm .03$ & $.77 \pm .02$ \\
\hline $\left.\begin{array}{ll}\tau & {[\mathrm{yr}}\end{array}\right]$ & $8.4 \pm .3$ & $7.8 \pm .2$ & $9.0 \pm .6$ & \\
\hline$\tau_{1} \quad[\mathrm{yr}]$ & & & $5.1 \pm .9$ & \\
\hline$h_{1}$ & & & $.141 \pm .016$ & \\
\hline$\gamma \quad\left[10^{6}\right.$ tons $\left./ \mathrm{yr}\right]$ & & $92 \pm 14$ & $92 \pm 14$ & $93 \pm 13$ \\
\hline$\delta \quad\left[10^{6}\right.$ tons $\left./ \mathrm{yr}\right]$ & & $189 \pm 28$ & $206 \pm 27$ & $213 \pm 25$ \\
\hline $\operatorname{SSR} \quad\left[10^{6} \text { tons }\right]^{2}$ & $1.321 \times 10^{6}$ & $6.548 \times 10^{5}$ & $5.637 \times 10^{5}$ & $5.637 \times 10^{5}$ \\
\hline$R^{2}$ & 9964 & .9982 & .9984 & .9984 \\
\hline
\end{tabular}


Table 5: Parameter estimates for temperature transfer function models of fossil fuel production. The parameter units are the same as in the previous table

\begin{tabular}{|c|c|c|c|c|c|}
\hline $\begin{array}{l}\text { Model } \\
n\end{array}$ & $\begin{array}{c}N=6 \\
11\end{array}$ & $\begin{array}{c}N=7 \\
12\end{array}$ & $\begin{array}{c}N=8 \\
13\end{array}$ & $\begin{array}{c}N=9 \\
14\end{array}$ & $\begin{array}{c}N=10 \\
15\end{array}$ \\
\hline$P_{0}$ & 187 & 183 & $186 \pm 5$ & 186 & 186 \\
\hline$\alpha$ & .0326 & .0327 & $.0327 \pm .0002$ & .0327 & .0327 \\
\hline$\beta$ & 0.75 & 0.76 & 0.78 & 0.77 & 0.76 \\
\hline$\gamma$ & 96 & 94 & $92 \pm 13$ & 93 & 94 \\
\hline$\delta$ & 240 & 229 & $215 \pm 27$ & 218 & 223 \\
\hline$\rho(\mathrm{SSR})$ & $6.044 \times 10^{5}$ & $5.717 \times 10^{5}$ & $5.341 \times 10^{5}$ & $5.326 \times 10^{5}$ & $5.294 \times 10^{5}$ \\
\hline$\rho_{1}$ & $8.278 \times 10^{5}$ & $6.044 \times 10^{5}$ & $5.717 \times 10^{5}$ & $5.341 \times 10^{5}$ & $5.326 \times 10^{5}$ \\
\hline$u$ & 40.658 & 6.225 & 7.608 & 0.315 & 0.629 \\
\hline$F_{0.95}(1,121-n)$ & 3.927 & 3.928 & 3.929 & 3.930 & 3.931 \\
\hline$R^{2}$ & .9983 & .9984 & .9985 & .9985 & .9985 \\
\hline
\end{tabular}




\section{Figure Captions}

Figure 1 The Mitchell-Brinkmann temperature record. The histogram ordinates are 5-year average Northern Hemispheric temperature variations from the 1880-1885 mean value. The smooth curve is an unweighted cubic smoothing spline fit with a smoothing constant of 0.05 .

Figure 2 Global fossil fuel production (1870-1974). The data points are measured yearly global totals expressed in megatons of carbon. The solid curve is a fit of the Rust-Kirk model (2.3), and the dashed curve is a fit of the unmodulated exponential (2.4).

Figure 3 Northern Hemisphere land temperature variations for the years 1851-1991. The data points are yearly average departures from the mean for the period 1951-1970. The dotted curve connecting the points is a cubic interpolating spline, and the solid curve is an optimal smoothing spline fit to the data.

Figure 4 Global fossil fuel production (1870-1990). The data points are measured yearly global totals expressed in megatons of carbon. The solid curve is the fit of the Rust-Kirk model (2.3) using the smoothing spline in Figure 3 for $T(t)$. The dashed curve is the fit of the exponential model (2.4) which is shown for comparison.

Figure 5 Global fossil fuel production (1870-1990). The solid curve is the fit of the lagged response model (4.2) [or the lagged average model (5.4)], using the smoothing spline representation for $T(t)$. The dashed curve is the fit of the exponential model (2.4) which is shown for comparison.

Figure 6 Global fossil fuel production (1870-1990). The curve is the fit of the lagged average (boxcar) model (5.4) using the interpolating spline representation for $T(t)$.

Figure 7 Residuals for the fit of the boxcar averaging model (6.1) or (5.4) using the interpolating spline representation for $T(t)$. The circles and dashed line segments mark the years 1929-1932 and 1944-1945.

Figure 8 Global fossil fuel production (1870-1990). The curve is the fit of the boxcar averaging model (7.5) which includes explicit terms for the Great Depression and World War II. 
Figure 9 Residuals (data - fit) for the boxcar averaging fit shown in Fig. 8. The circles and dashed line segments mark the years 1929-1932 and 1944-1945.

Figure 10 Global fossil fuel production (1870-1990). The curve is the fit of an exponential augmented with terms for the Great Depression and World War II, i.e., the model (7.7).

Figure 11 Global fossil fuel production (1870-1990). The curve is the fit of the model obtained by augmenting the Rust-Kirk model with terms for the Great Depression and World War II, i.e., the model (7.8), and using the optimal smoothing spline representation of $T(t)$.

Figure 12 Global fossil fuel production (1870-1990). The curve is the fit of the augmented boxcar averaging model (7.5) using the optimal smoothing spline representation of $T(t)$.

Figure 13 The relationship between the temperature record $T\left(t^{\prime}\right)$ and the temperature averaging function $w\left(t^{\prime \prime} ; \tau\right)=w\left(t^{\prime}-t ; \tau\right)$.

Figure 14 The quadrilateral averaging function.

Figure 15 Global fossil fuel production (1870-1990). The curve is the fit of the quadrilateral averaging model (9.4) using the interpolating spline representation of $T(t)$.

Figure 16 Temperature averaging functions estimated from fits to the fossil fuel production record (1870-1990). The dashed lines represent the quadrilateral window from the fit shown in Fig. 15 and the solid lines represent the boxcar window obtained from the fit in Fig. 8.

Figure 17 Discrete point estimates of the temperature transfer function for the values $\tau=7,8,9,10$, and 11 . The discrete points are joined by straight line segments, but are indicated by plotted symbols only in the case of $\tau=9$ where they are indicated by small circles with $\pm 1 \sigma$ error bars attached.

Figure 18 Estimates of the temperature averaging function. The two dashed curves represent the boxcar and quadrilateral estimates given in columns 2 and 3 of Table 4 . The circles are the the estimated discrete ordinates for the $\tau=9$ transfer function, with the error bars indicating the $\pm 1 \sigma$ uncertainties. The solid curve is the optimal smoothing spline fit to those ordinates, renormalized to satisfy the unit area constraint (8.3). 
Figure 19 Global fossil fuel production (1870-1990). The curve is the fit of the $N=8(\tau=9)$ transfer function model (10.12).

Figure 20 Distribution of SSR values for the fits of the $N=8(\tau=9)$ transfer function model (10.12) using 10,000 randomly chosen temperature timeseries.

Figure 21 Global fossil fuel production (1870-1990). The solid curve is the fit of the lagged response model (12.1), and the dashed curve is the best fitting unmodulated exponential with the same rate constant $\alpha$ and the same perturbations for the Great Depression and World War II.

Figure 22 Residuals (data - model) for the fits given in Figure 21.

Figure 23 The temperature modulation and the perturbations for the Great Depression and World War II. The solid curve is the difference of the two model predictions plotted in Figure 21, and the dashed curves are the drops used in both of those models.

Figure 24 The temperature modulation and the perturbations for the Great Depression and World War II expressed as a fraction of the total production in the same year.

Figure 25 Three future temperature scenarios. The data points and the smoothing spline fit to them are the same as those in Figure 3.

Figure 26 Fossil fuel production predictions for the three temperature scenarios given in Figure 25. The data points and the curve through them are the same as those in Figure 21. 
North. Hemisph. Land Temp.

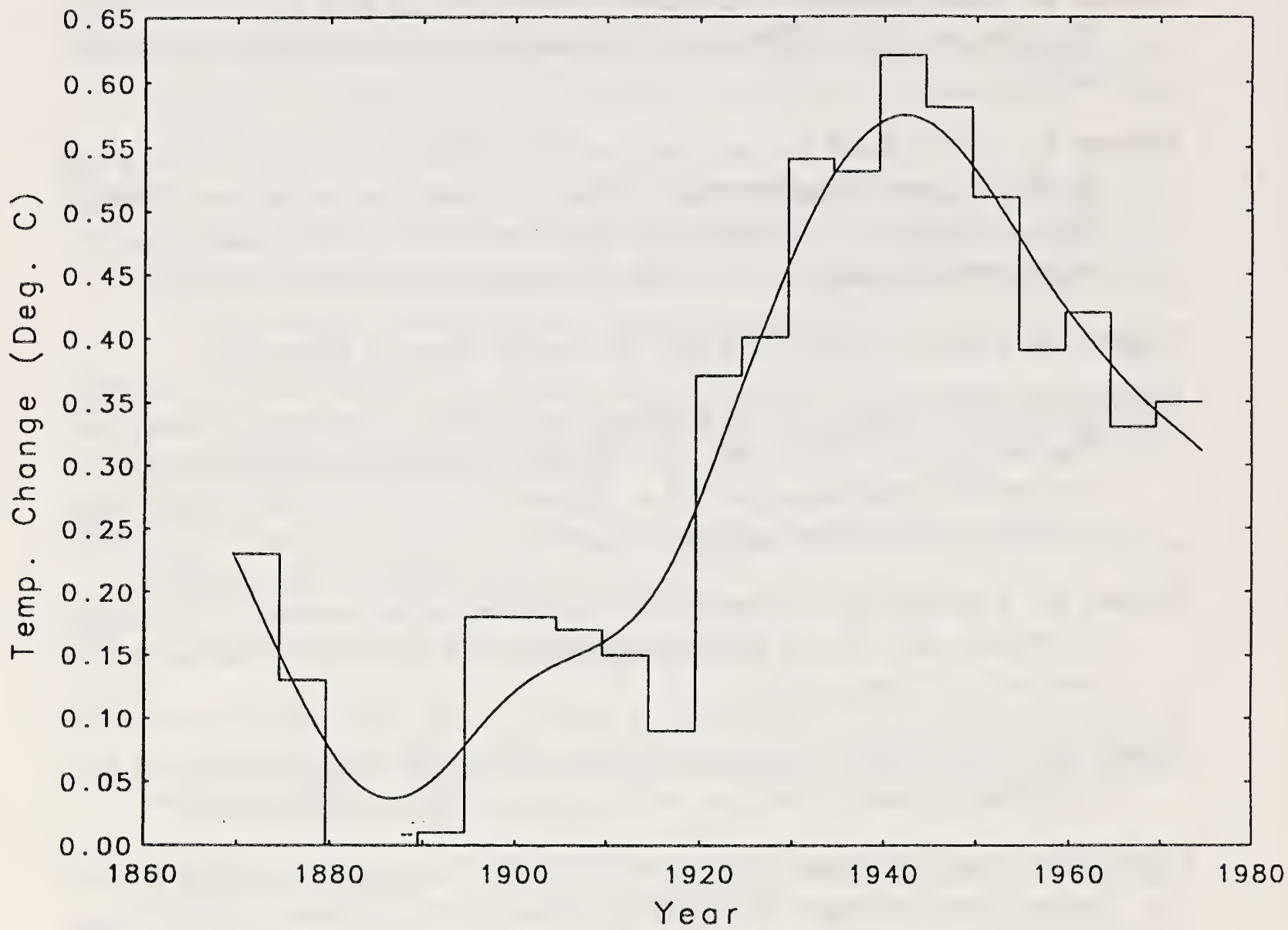

Figure 1: 


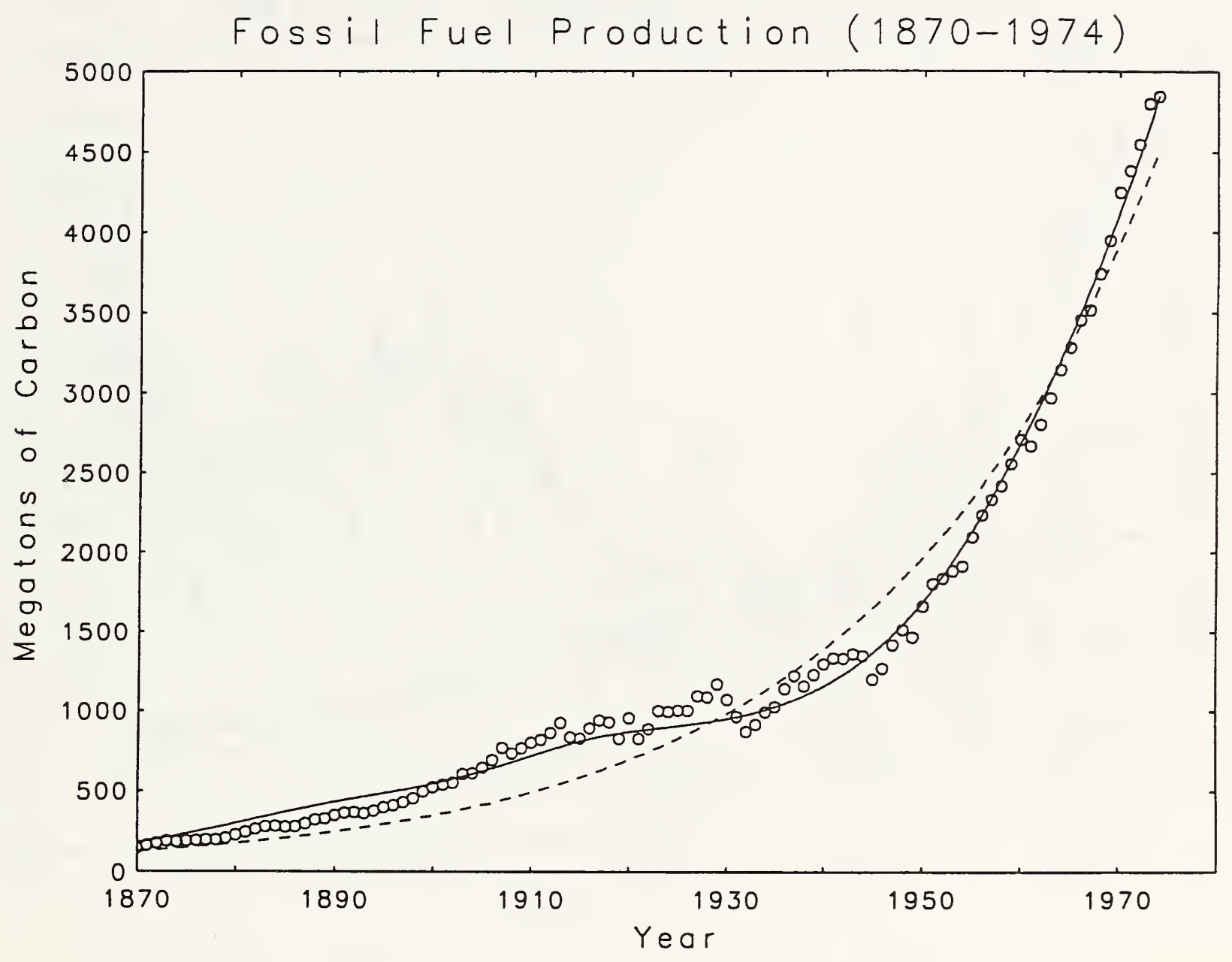

Figure 2: 


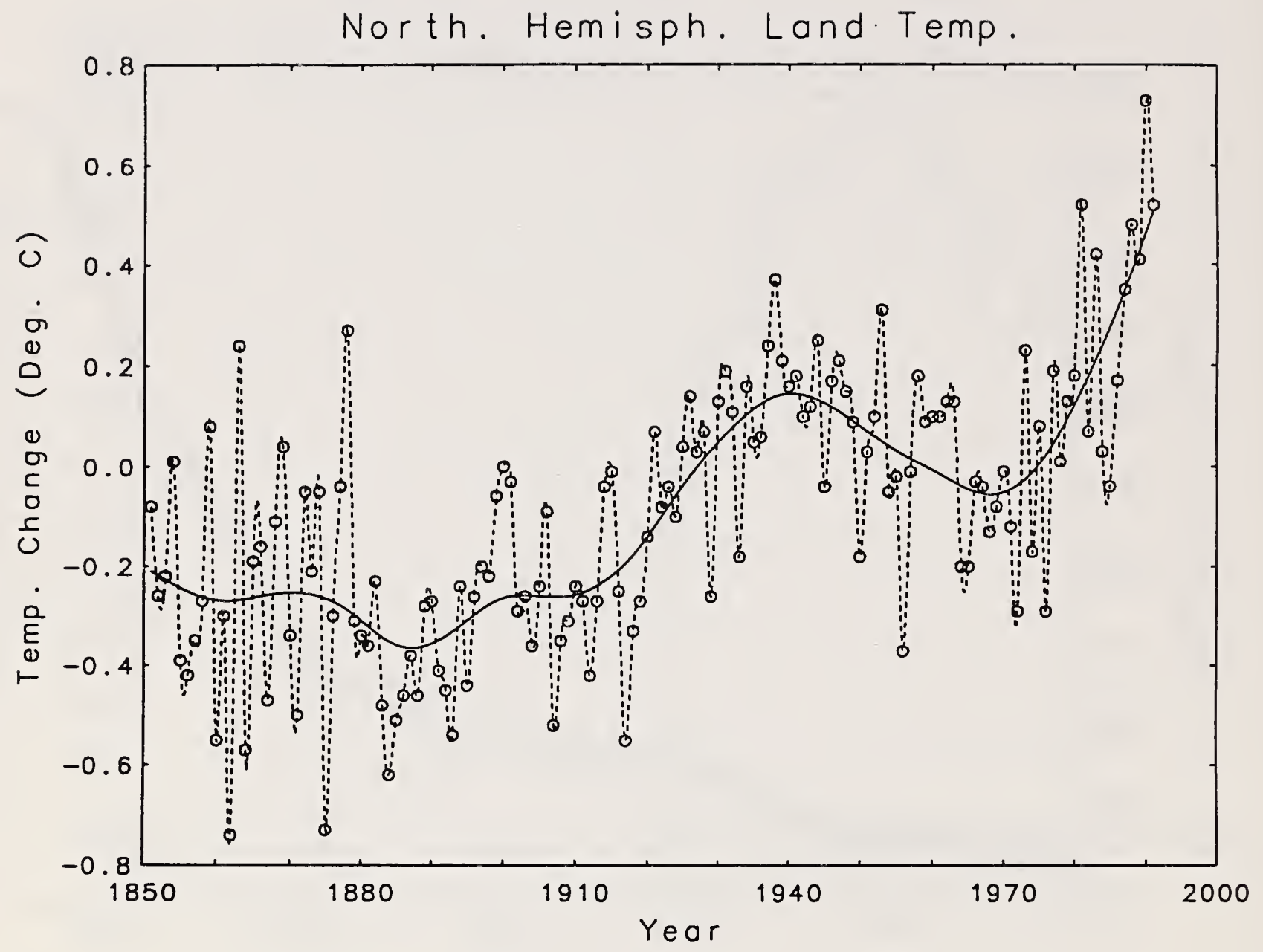

Figure 3: 


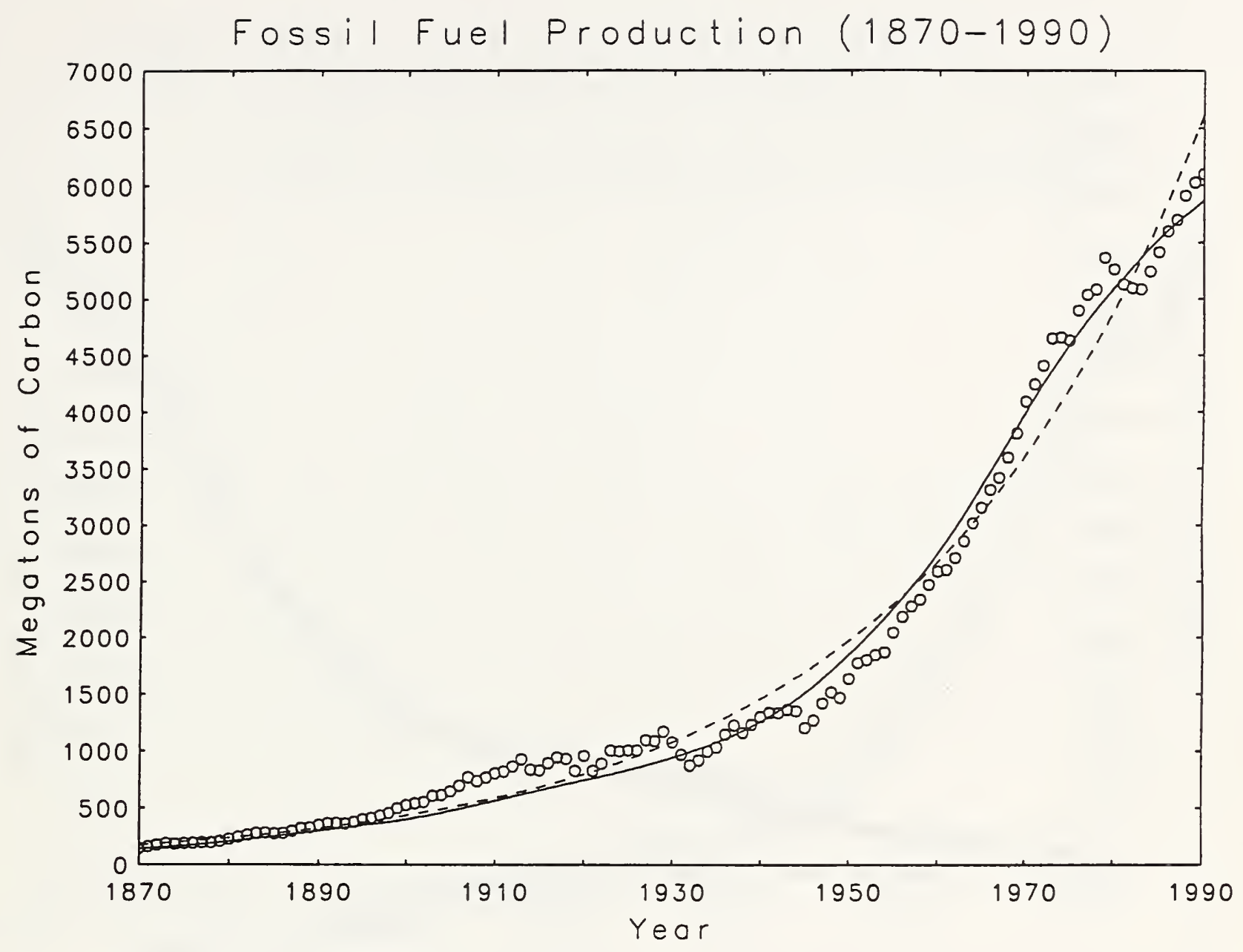

Figure 4: 


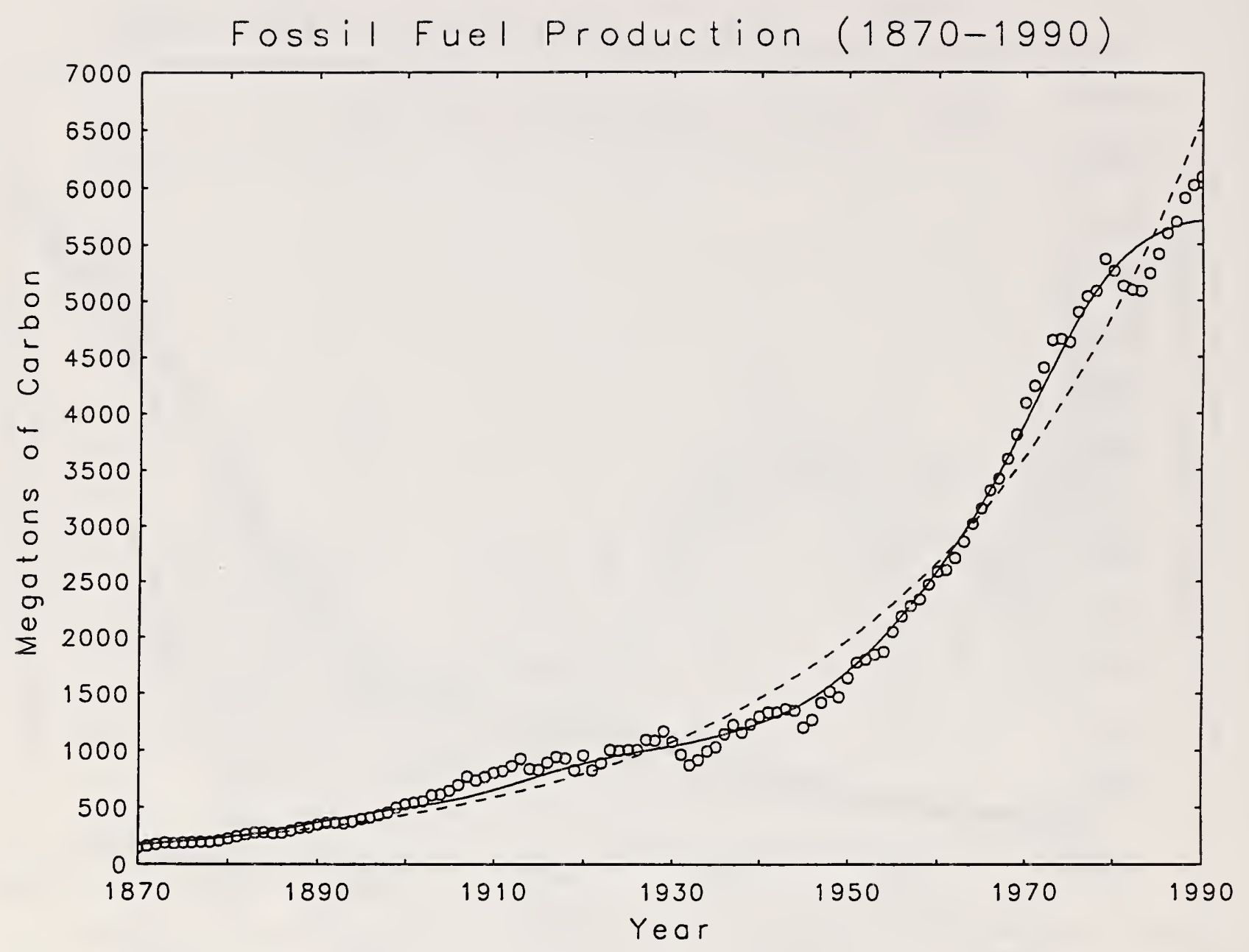

Figure 5: 


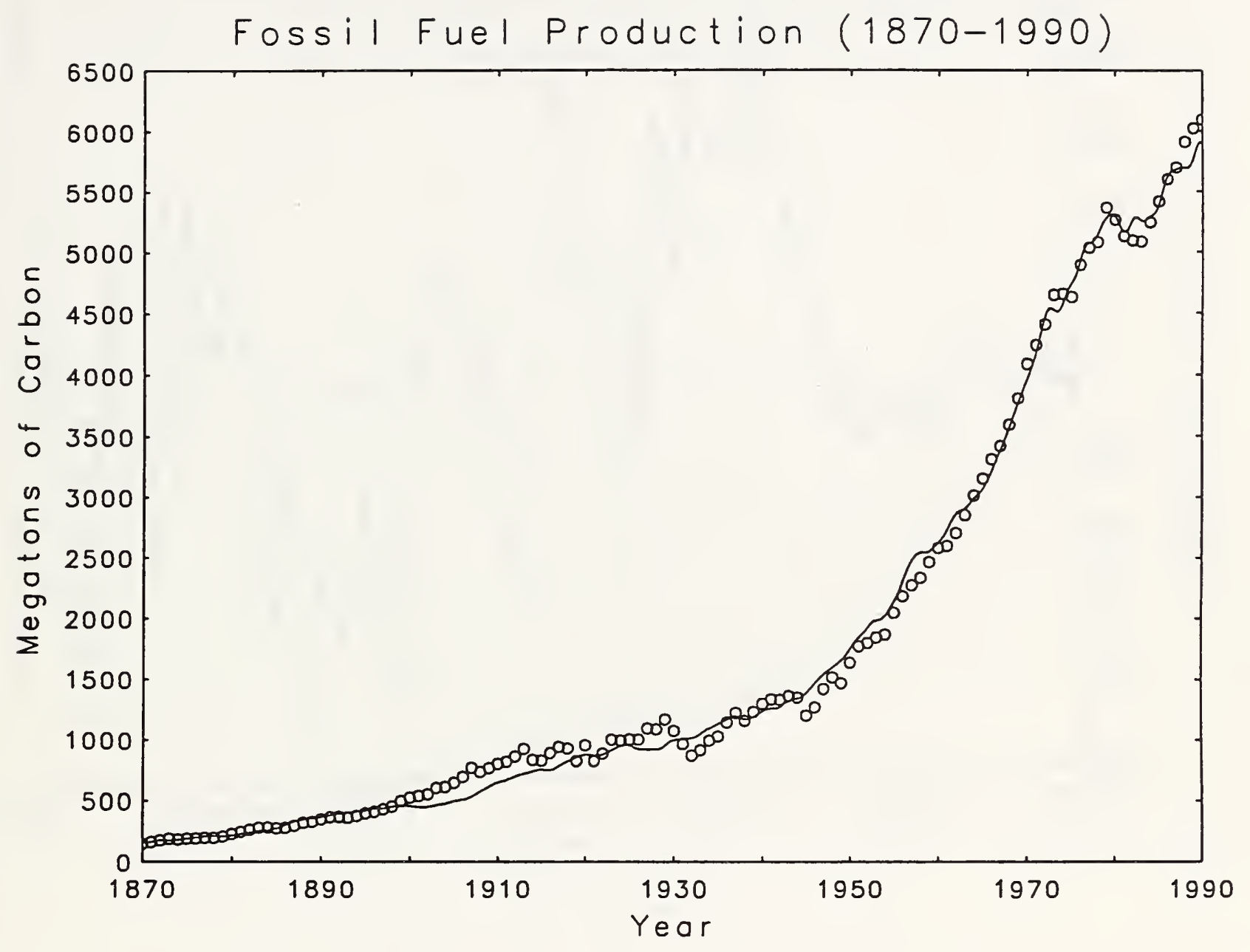

Figure 6: 


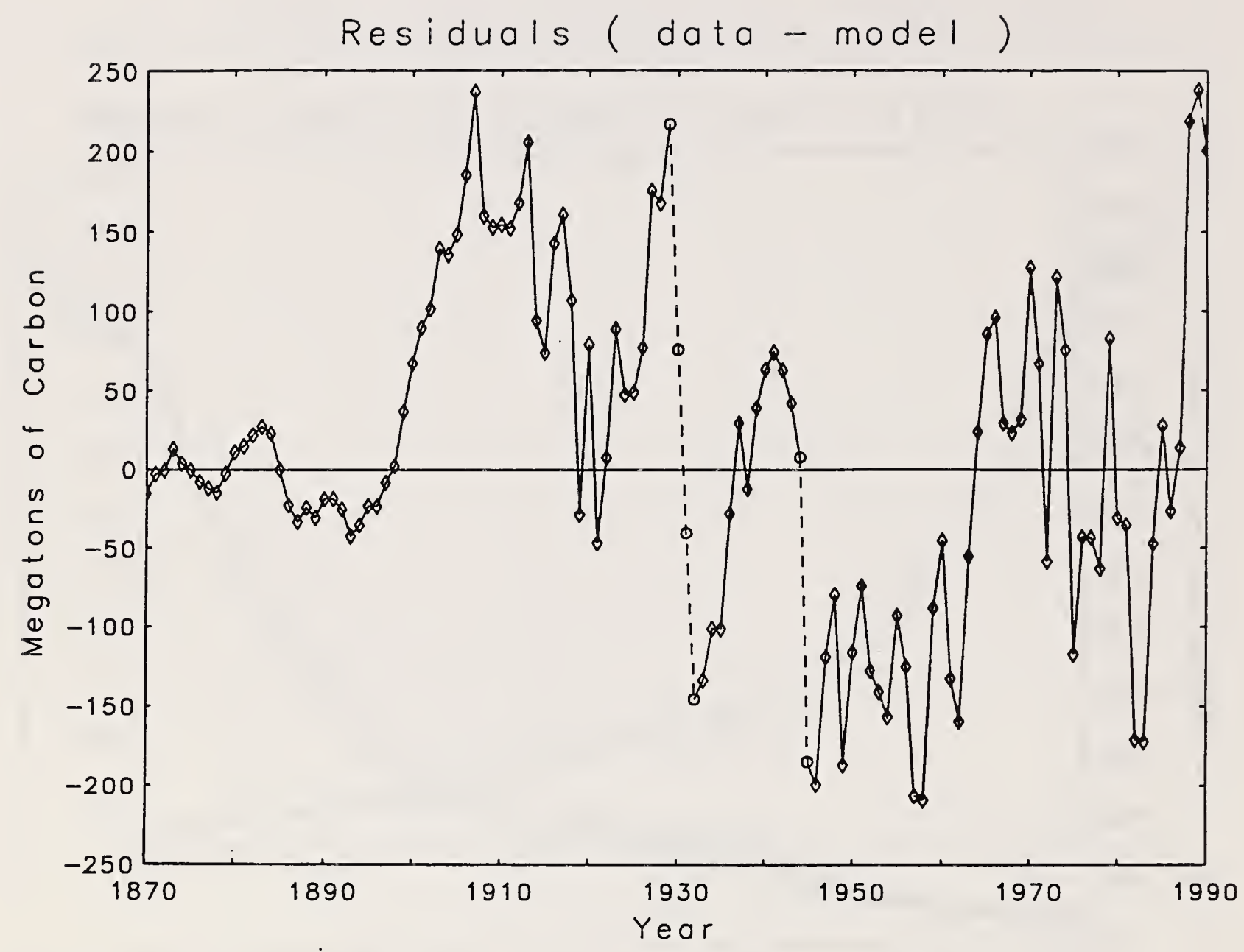

Figure 7: 


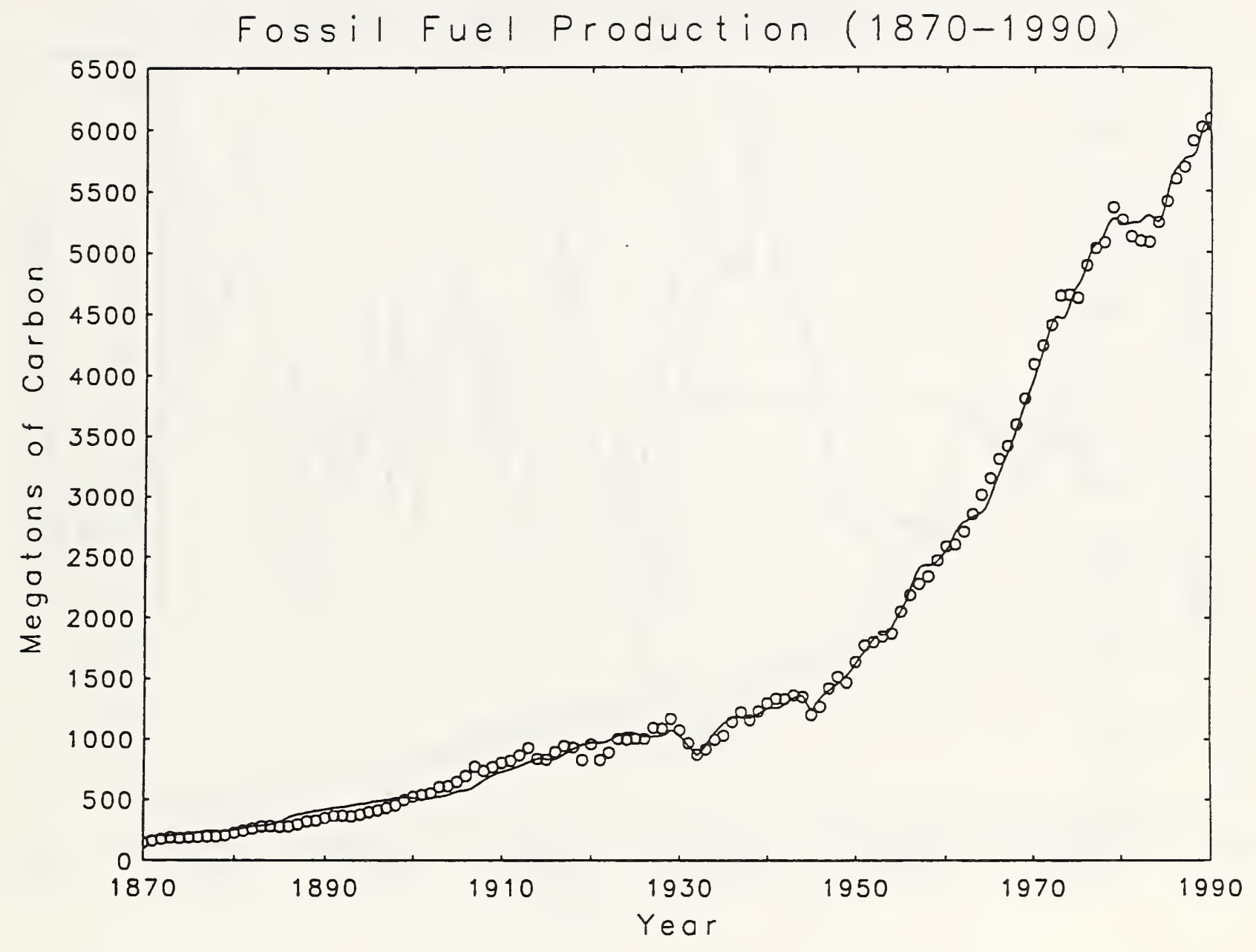

Figure 8: 


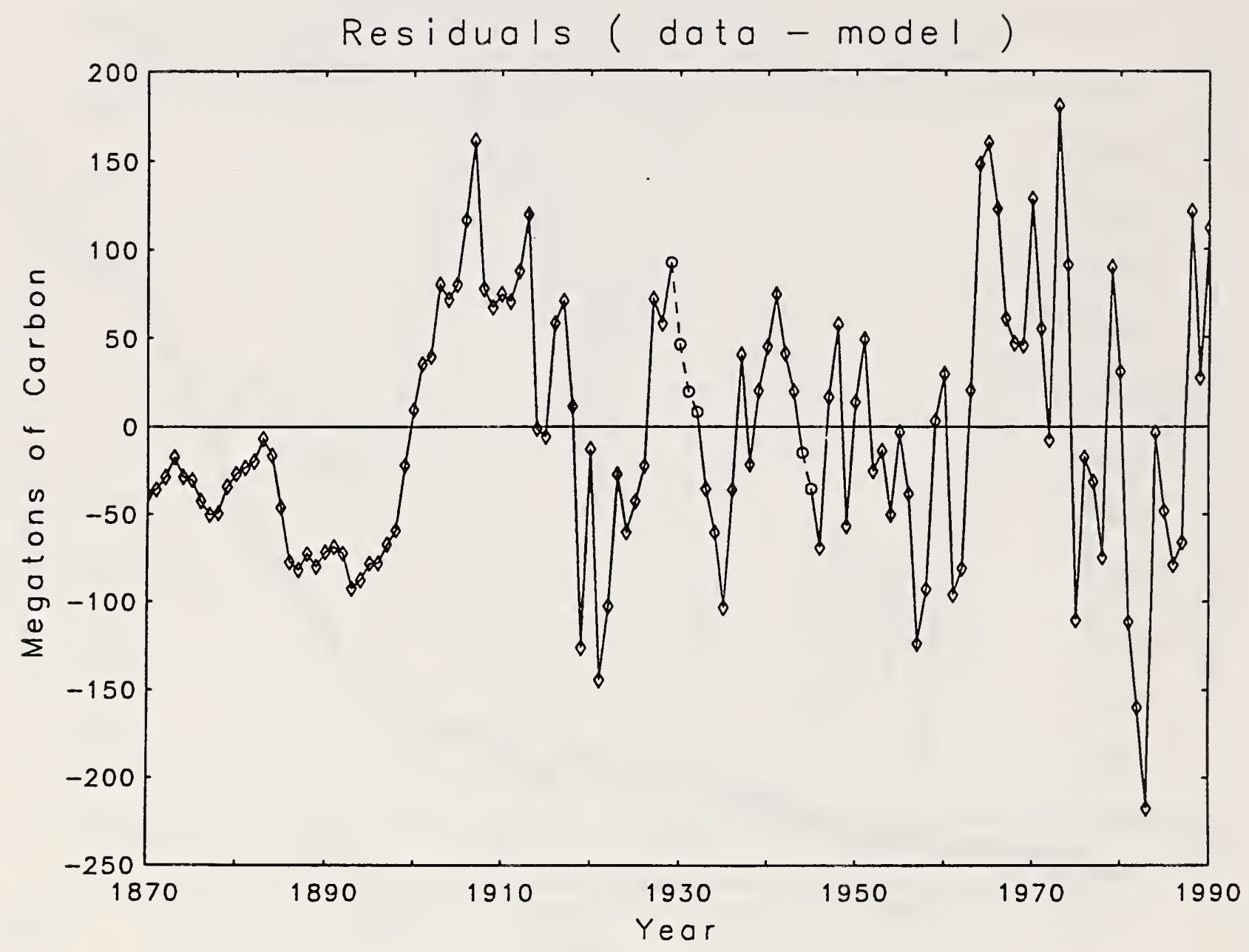

Figure 9: 


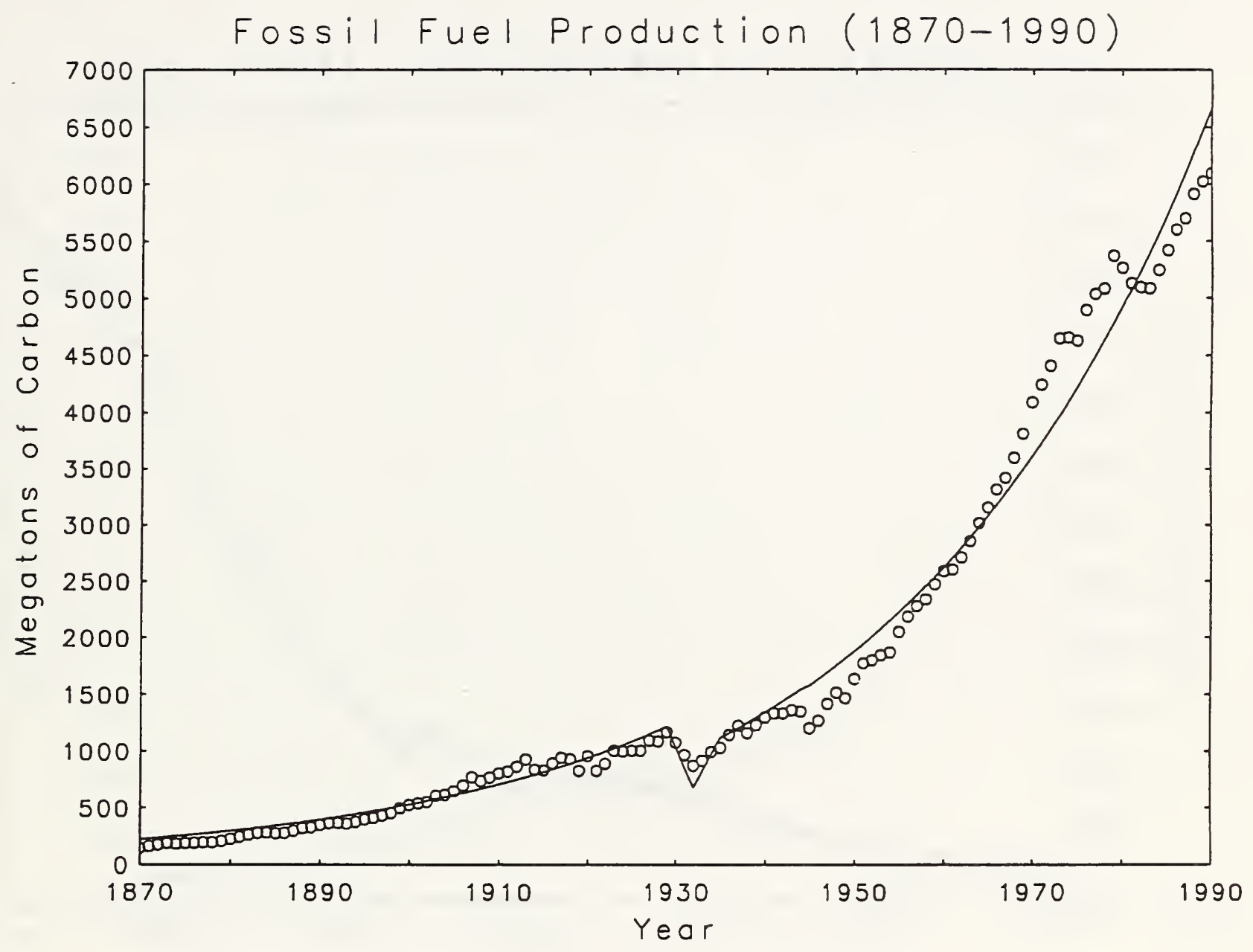

Figure 10: 


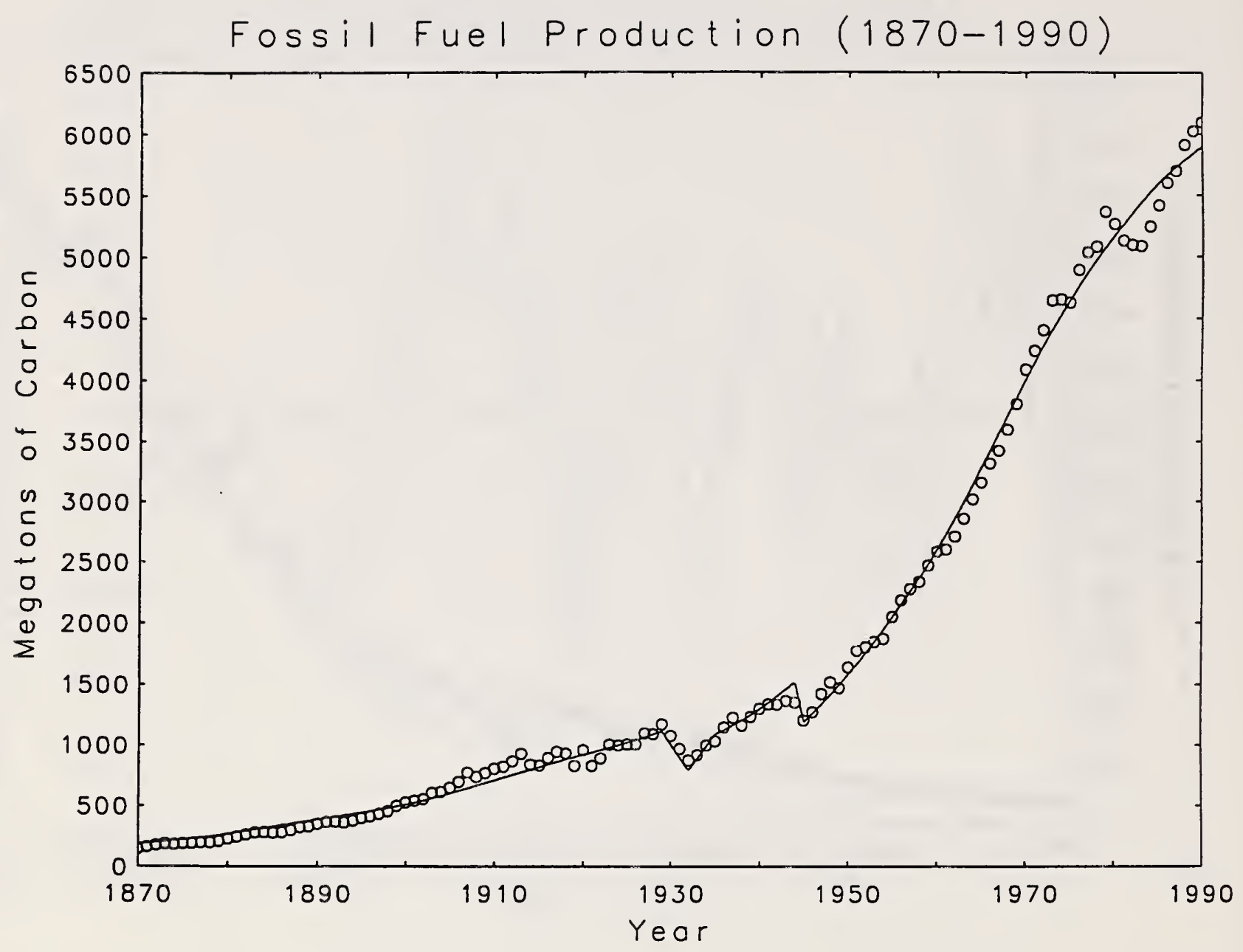

Figure 11: 


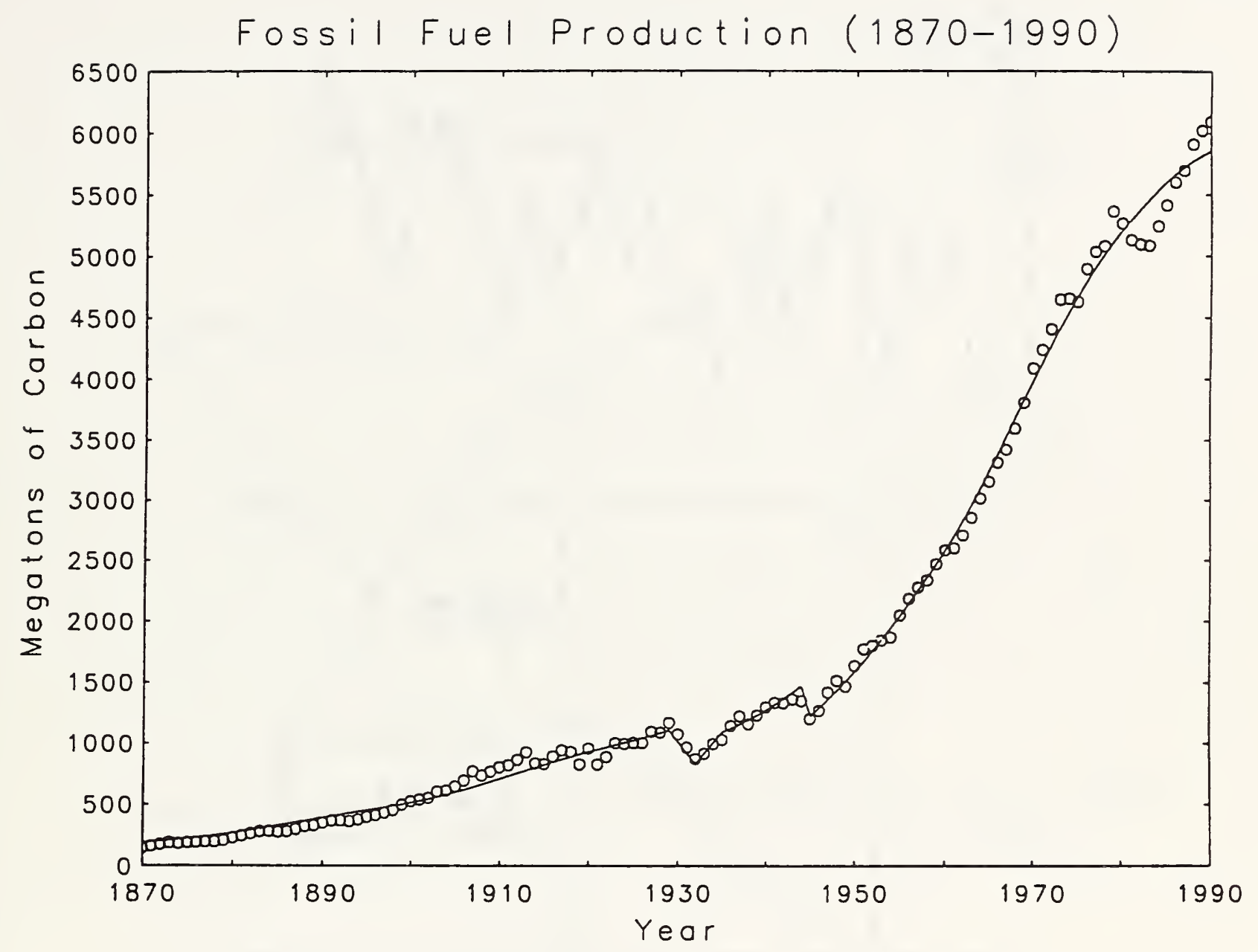

Figure 12: 


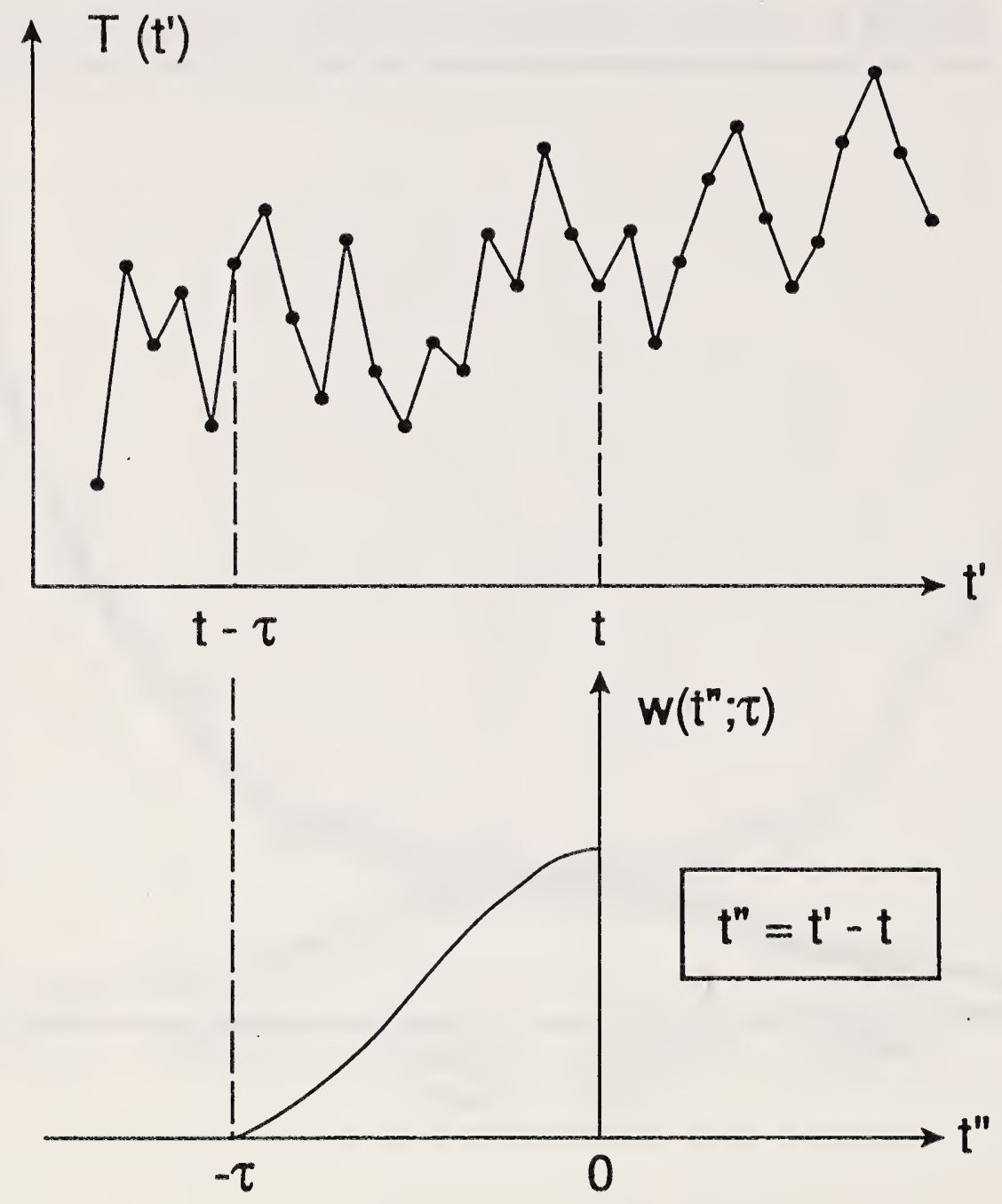

Figure 13: 


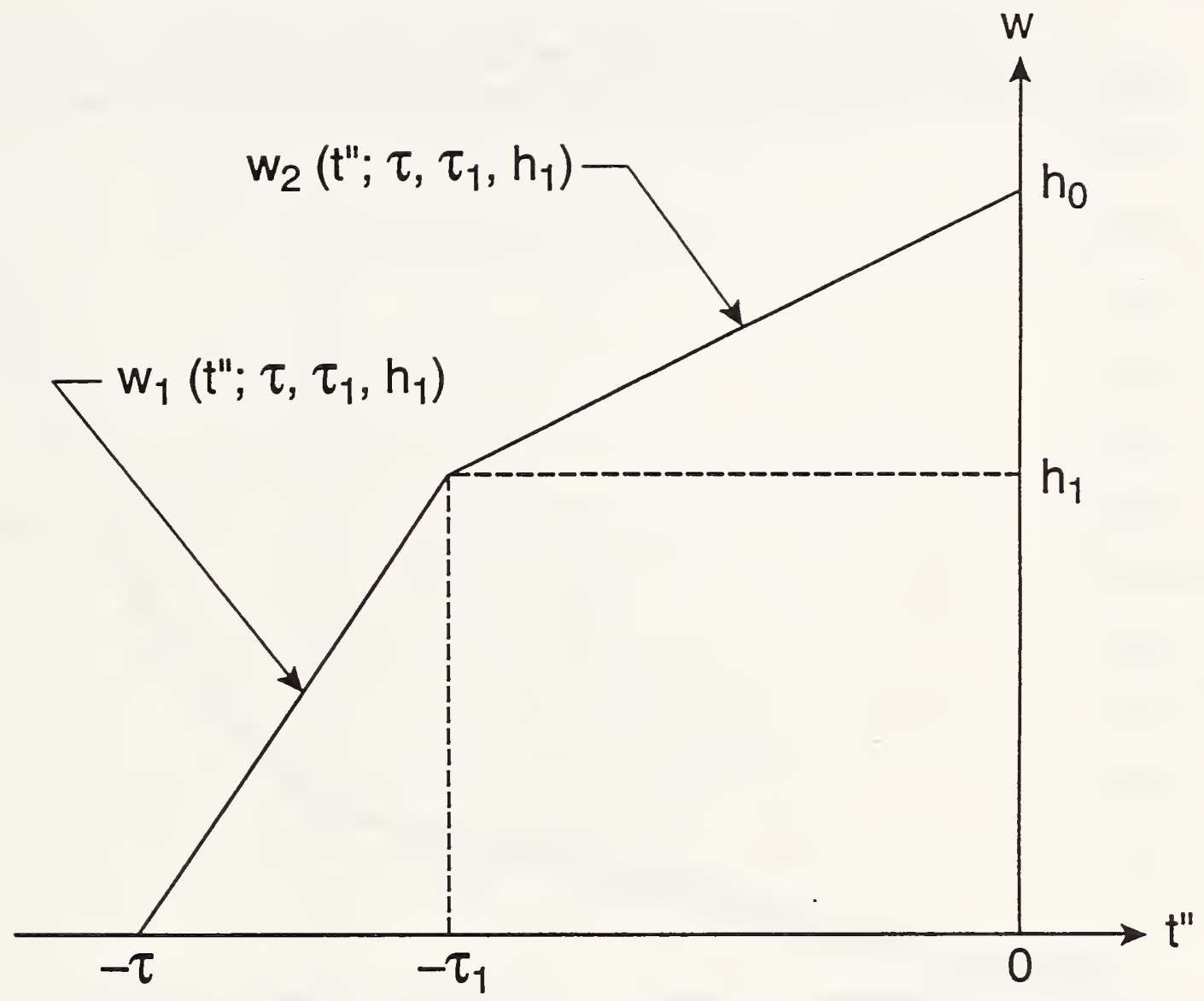

Figure 14: 


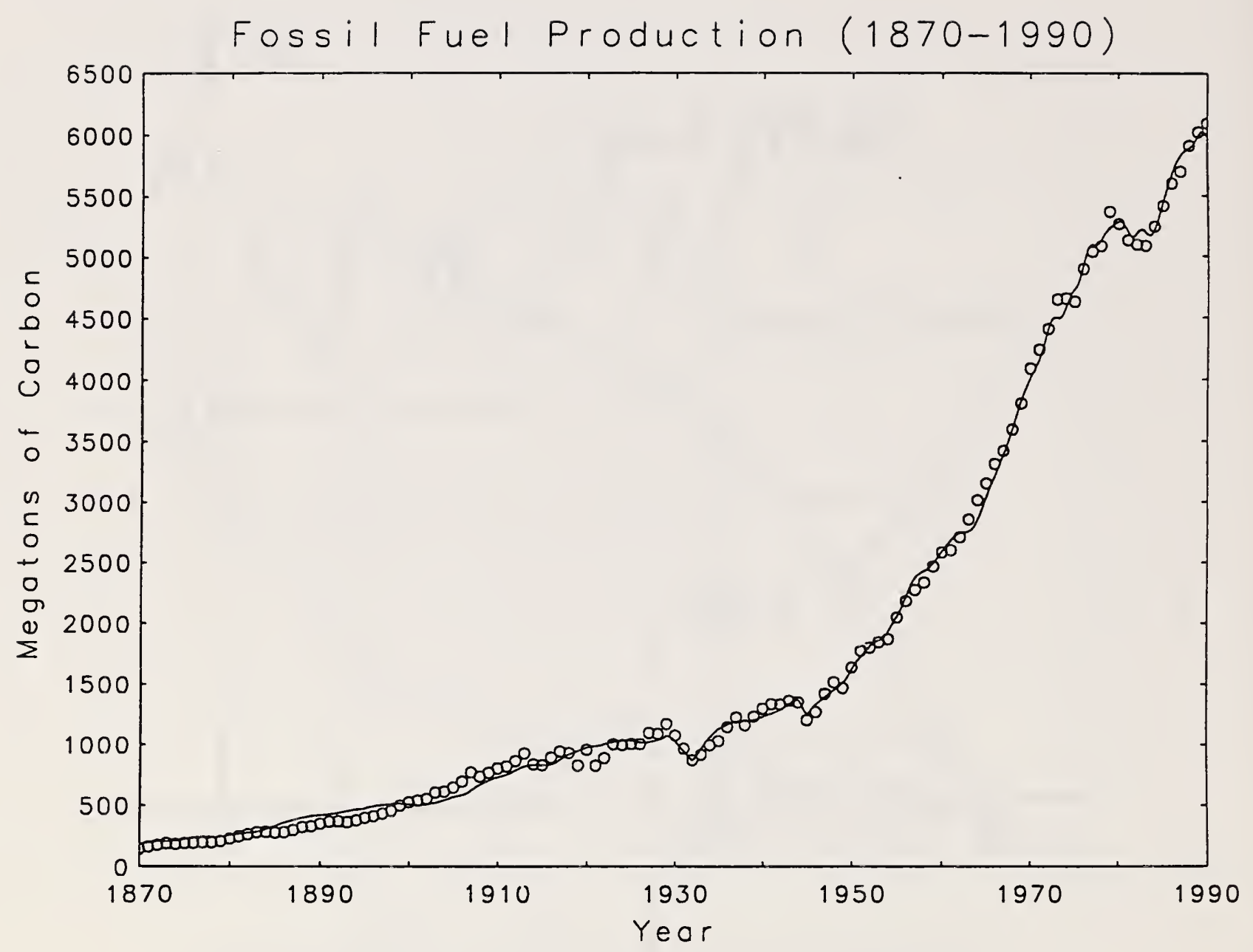

Figure 15: 


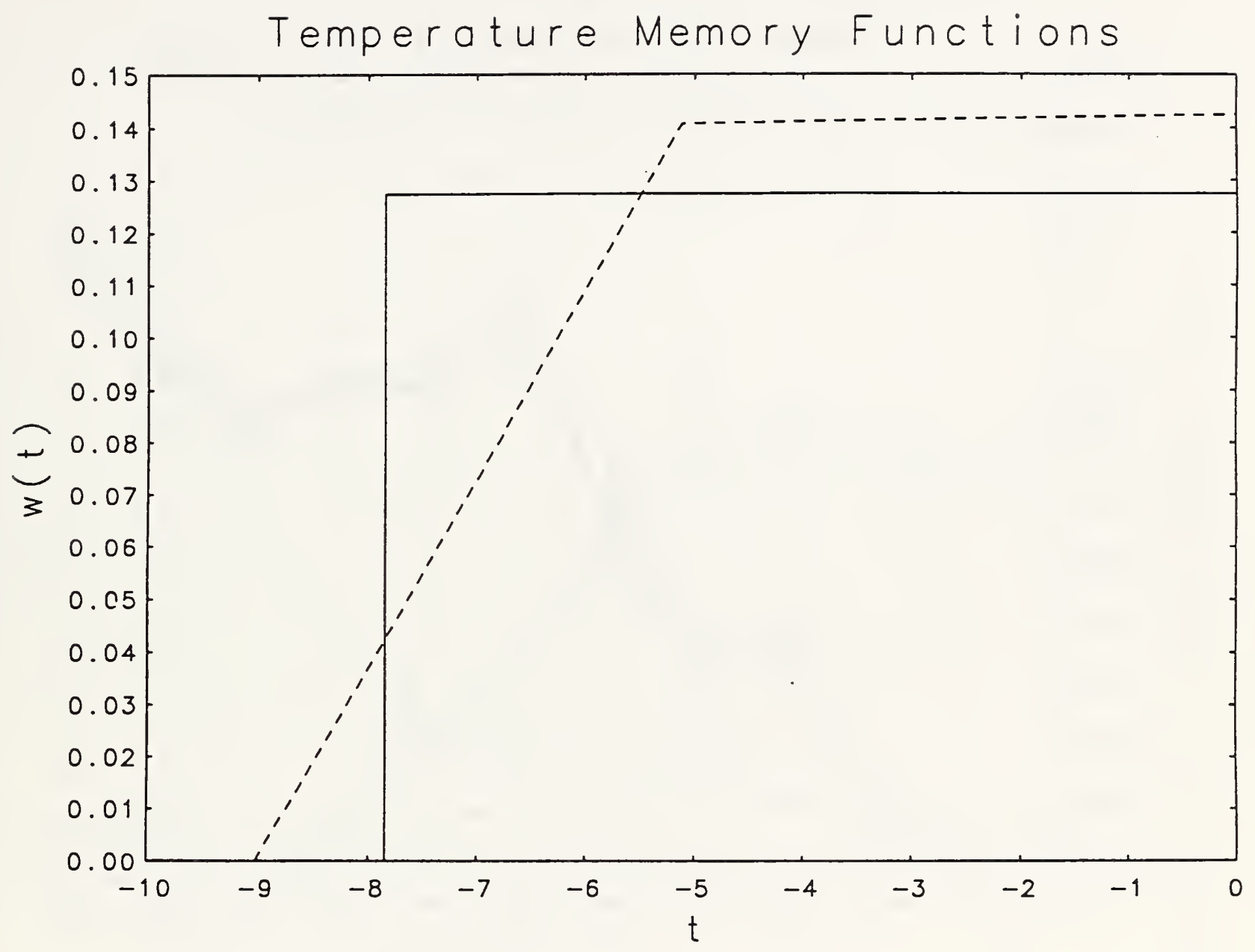

Figure 16: 


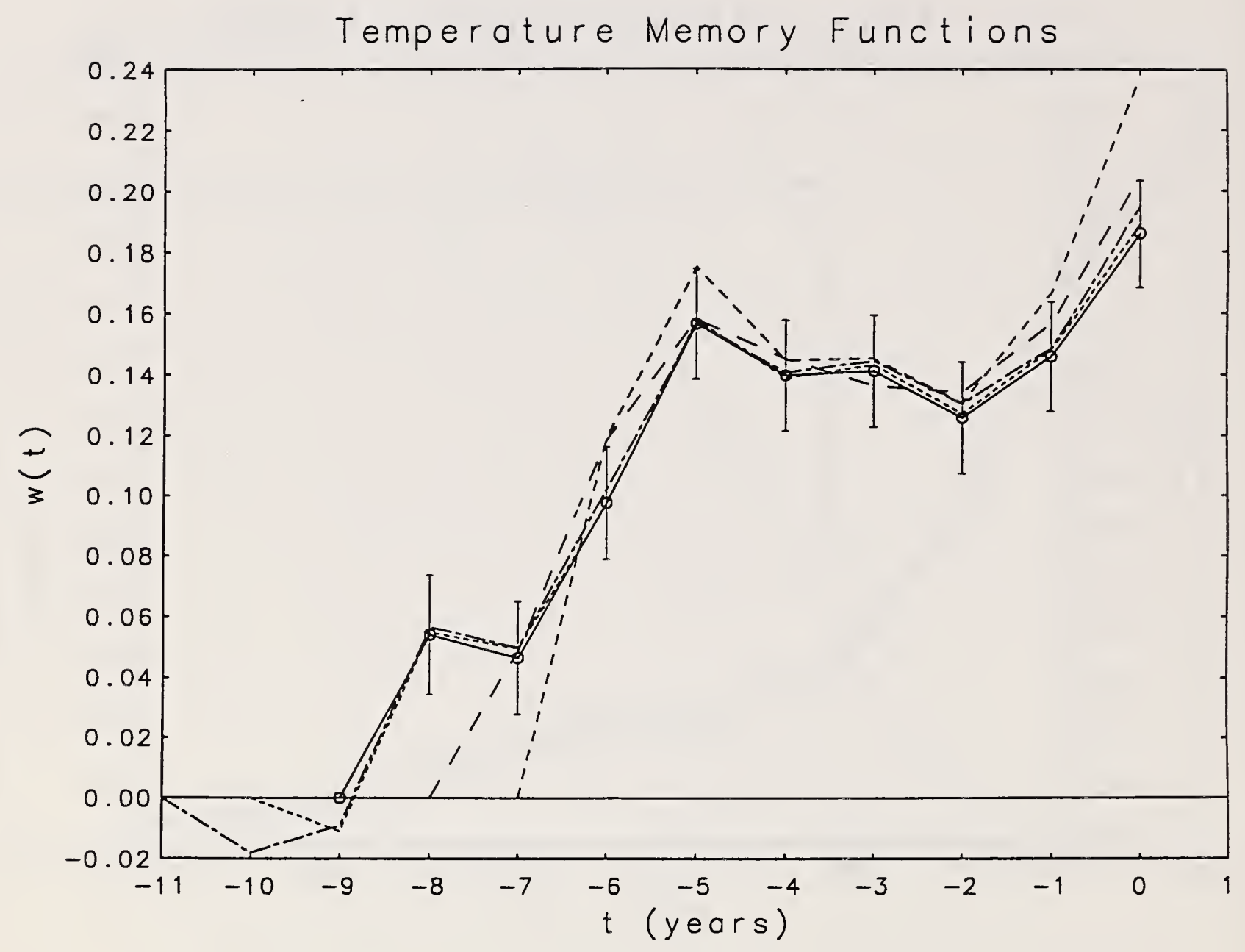

Figure 17: 


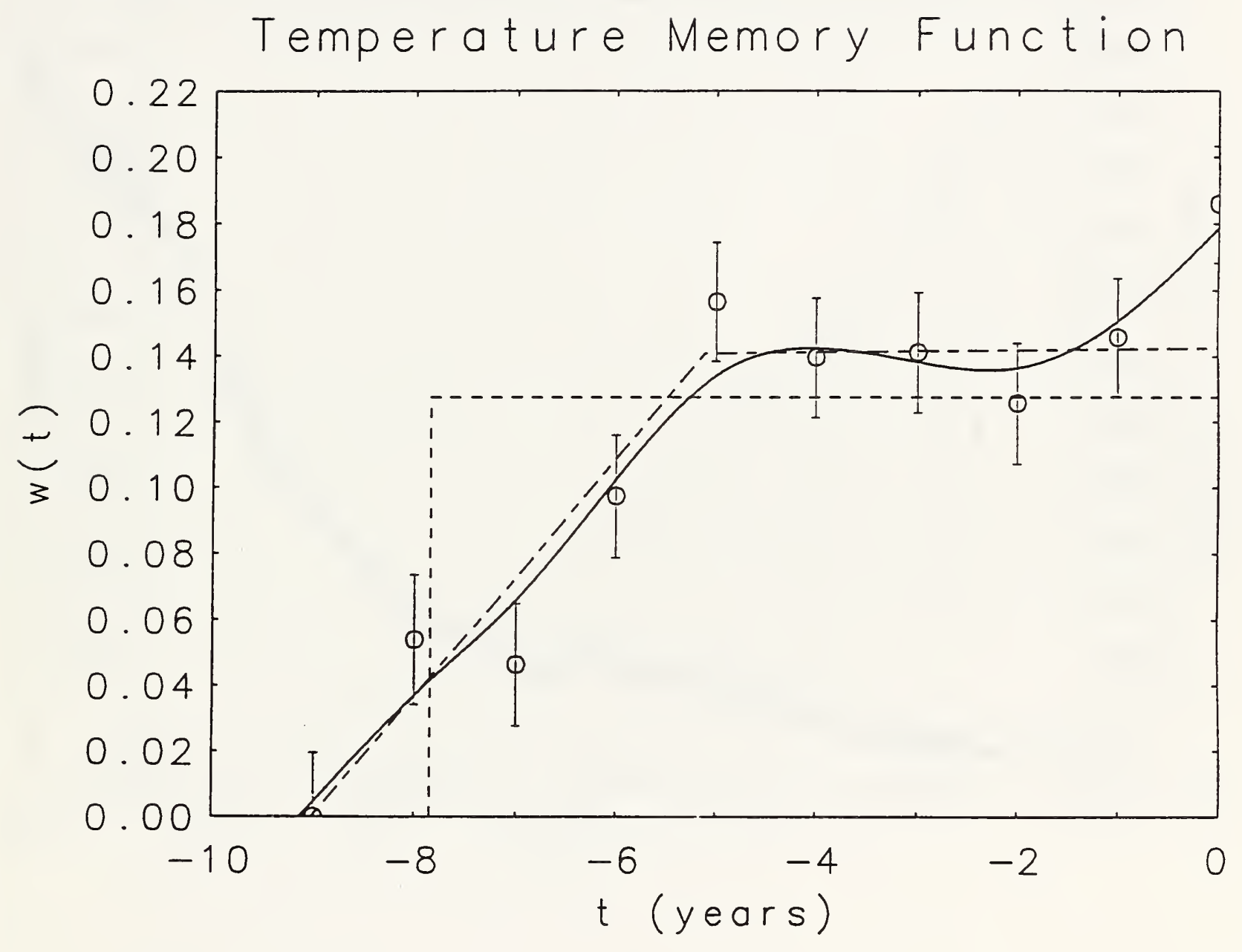

Figure 18: 


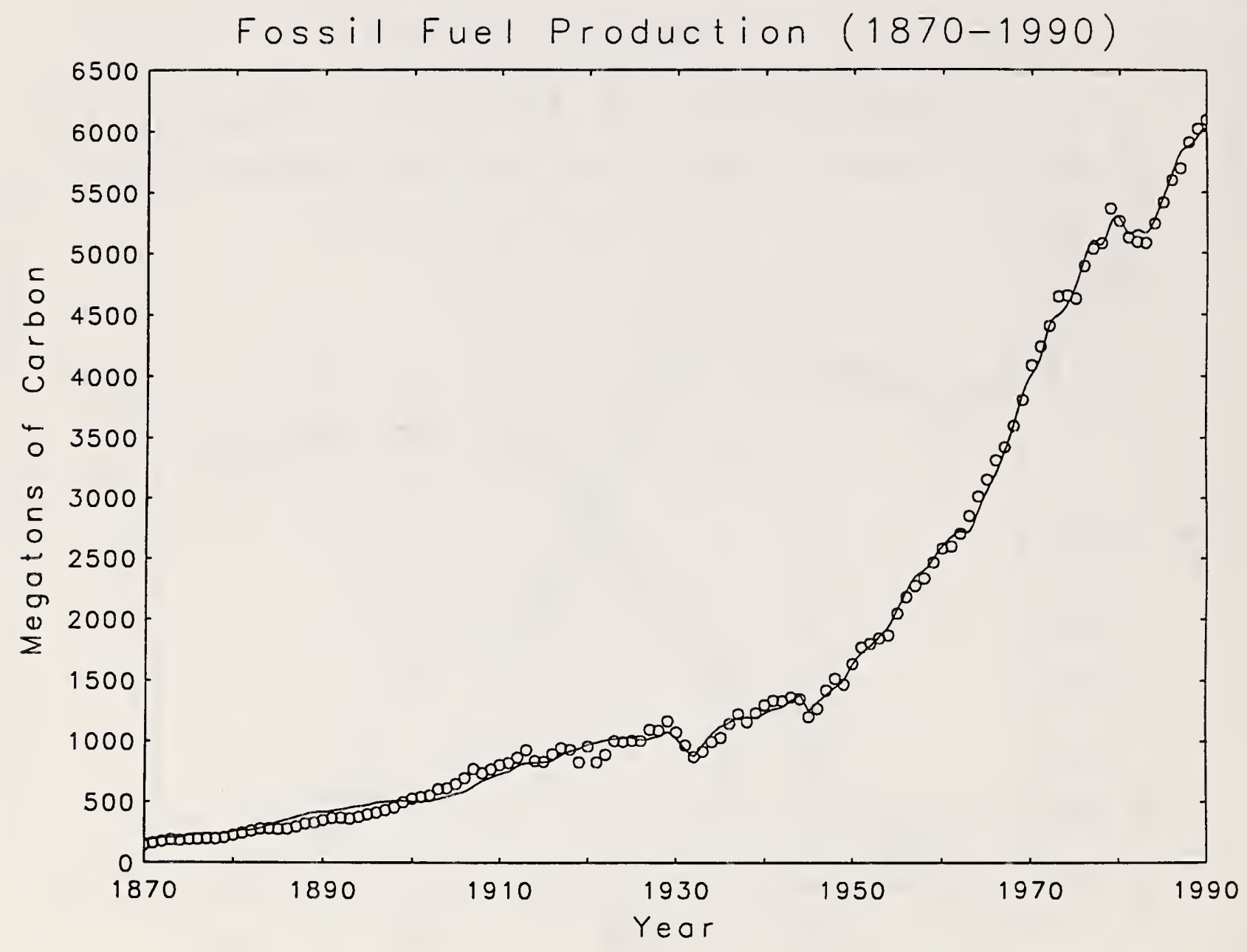

Figure 19: 


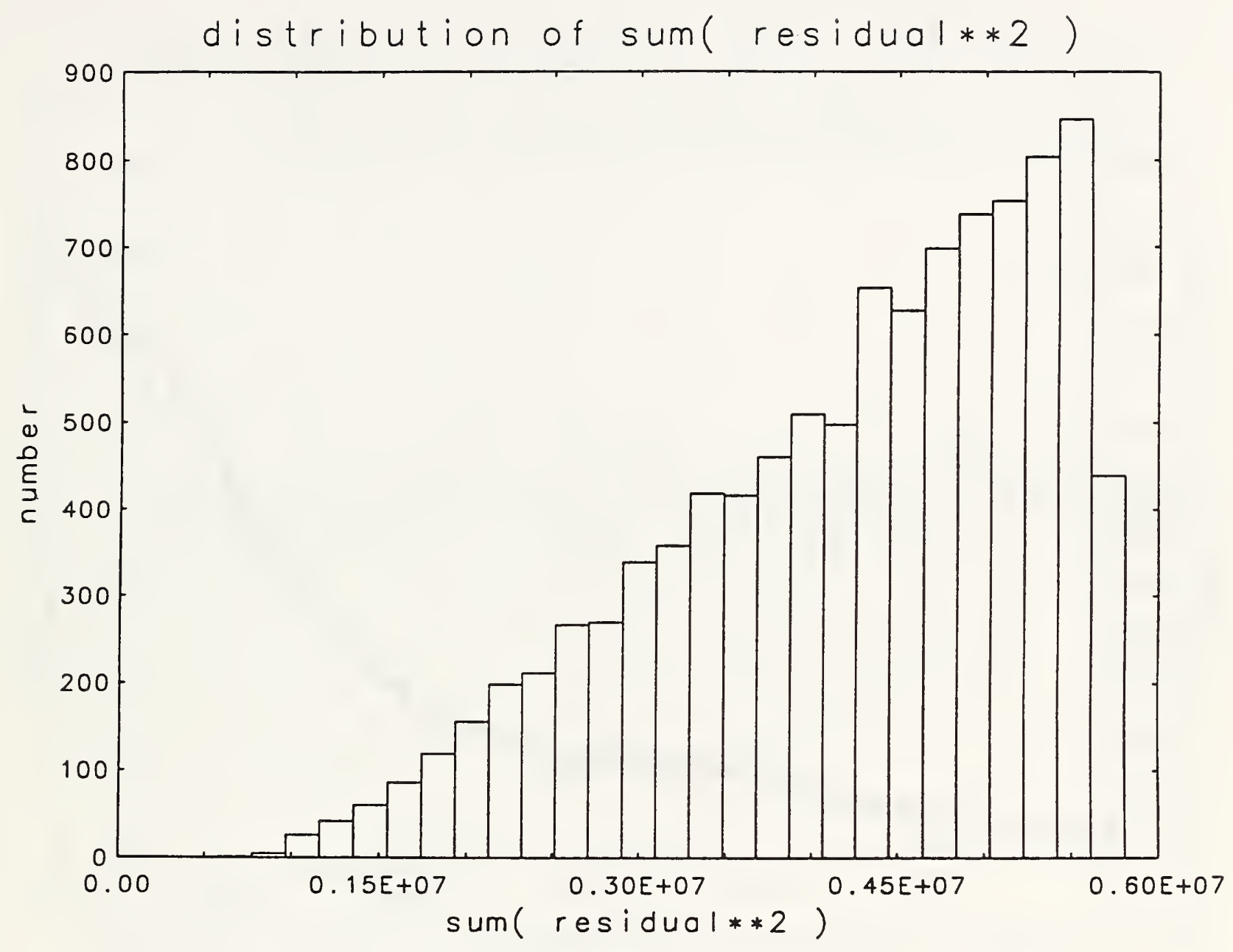

Figure 20: 


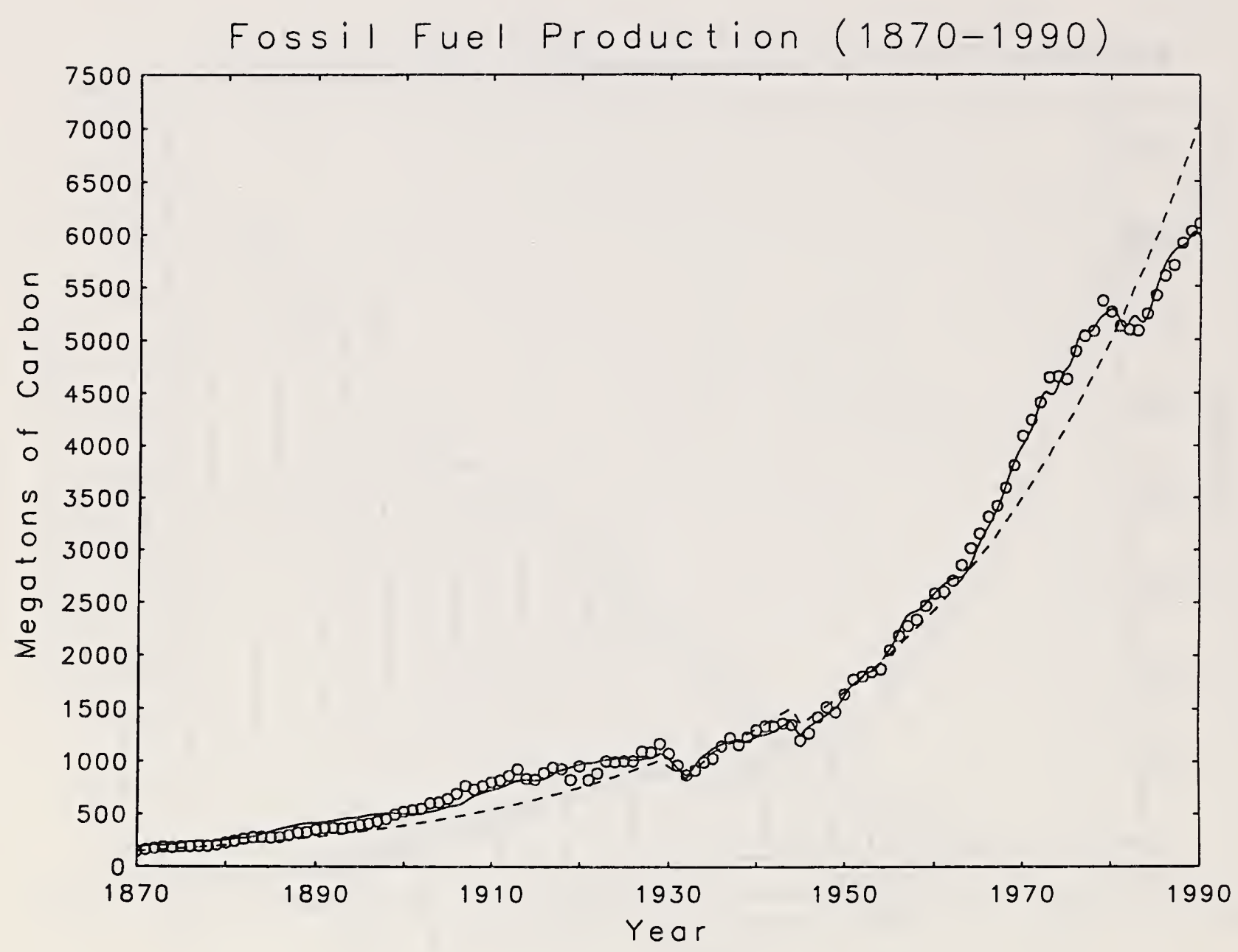

Figure 21: 


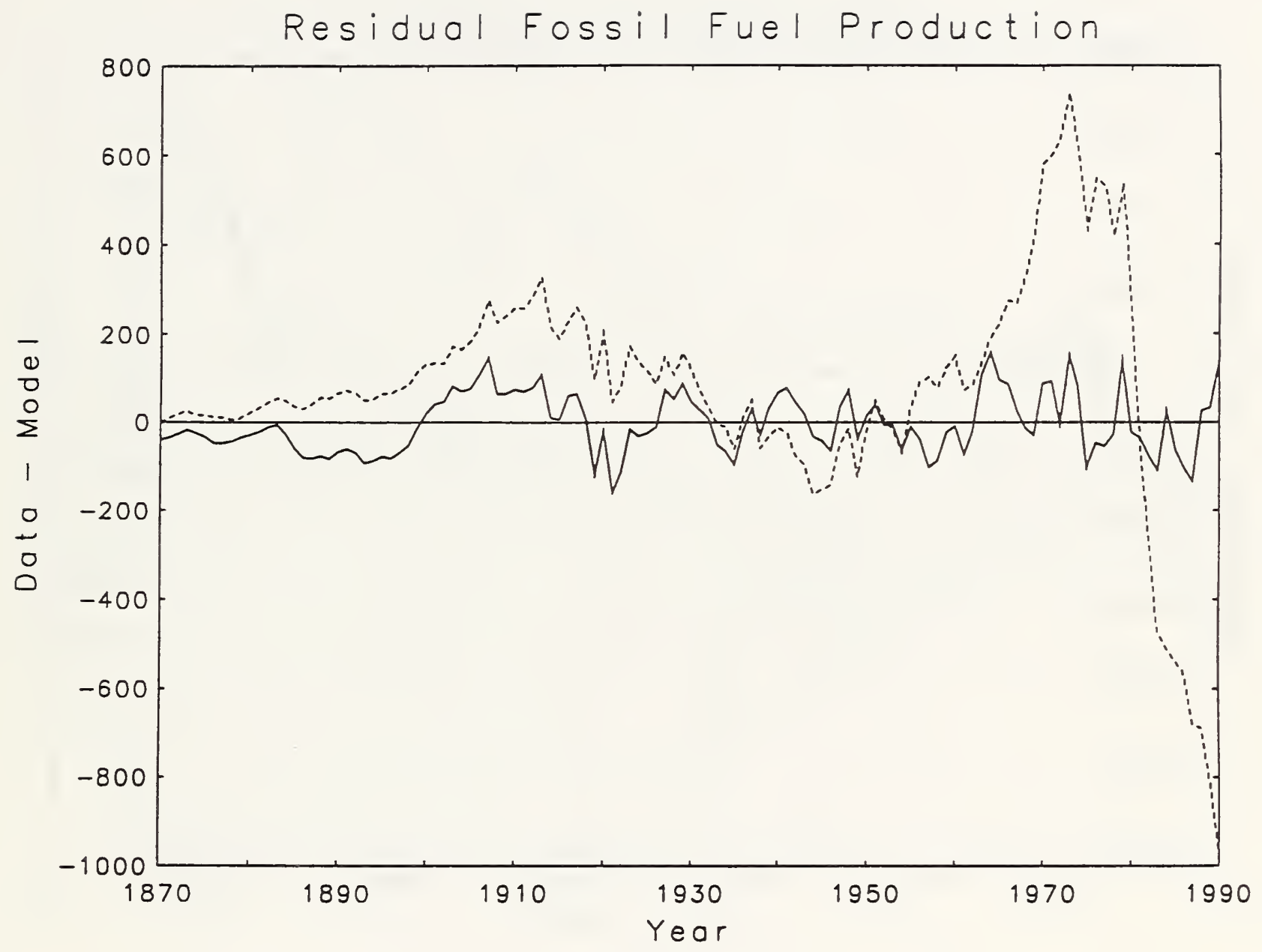

Figure 22: 


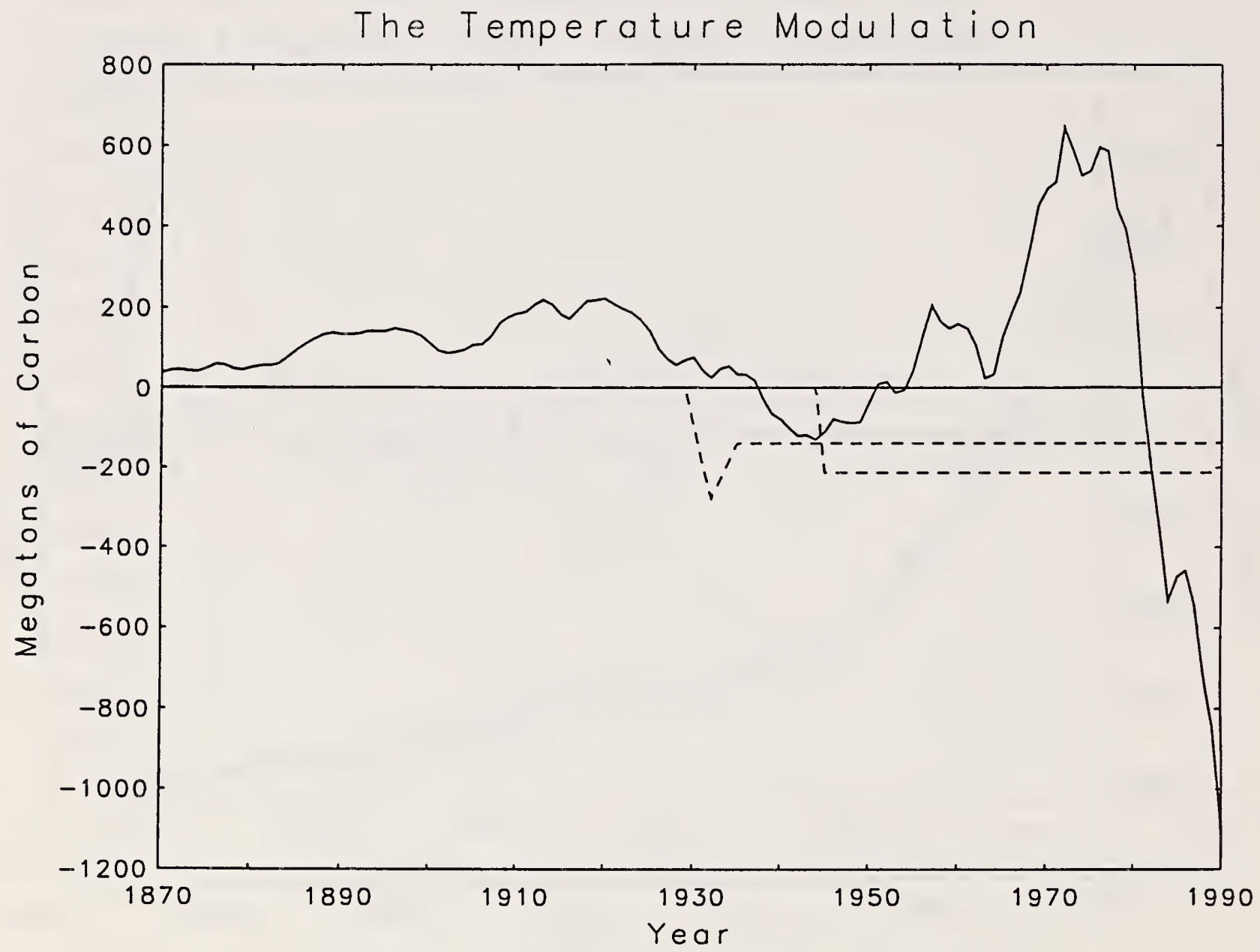

Figure 23: 


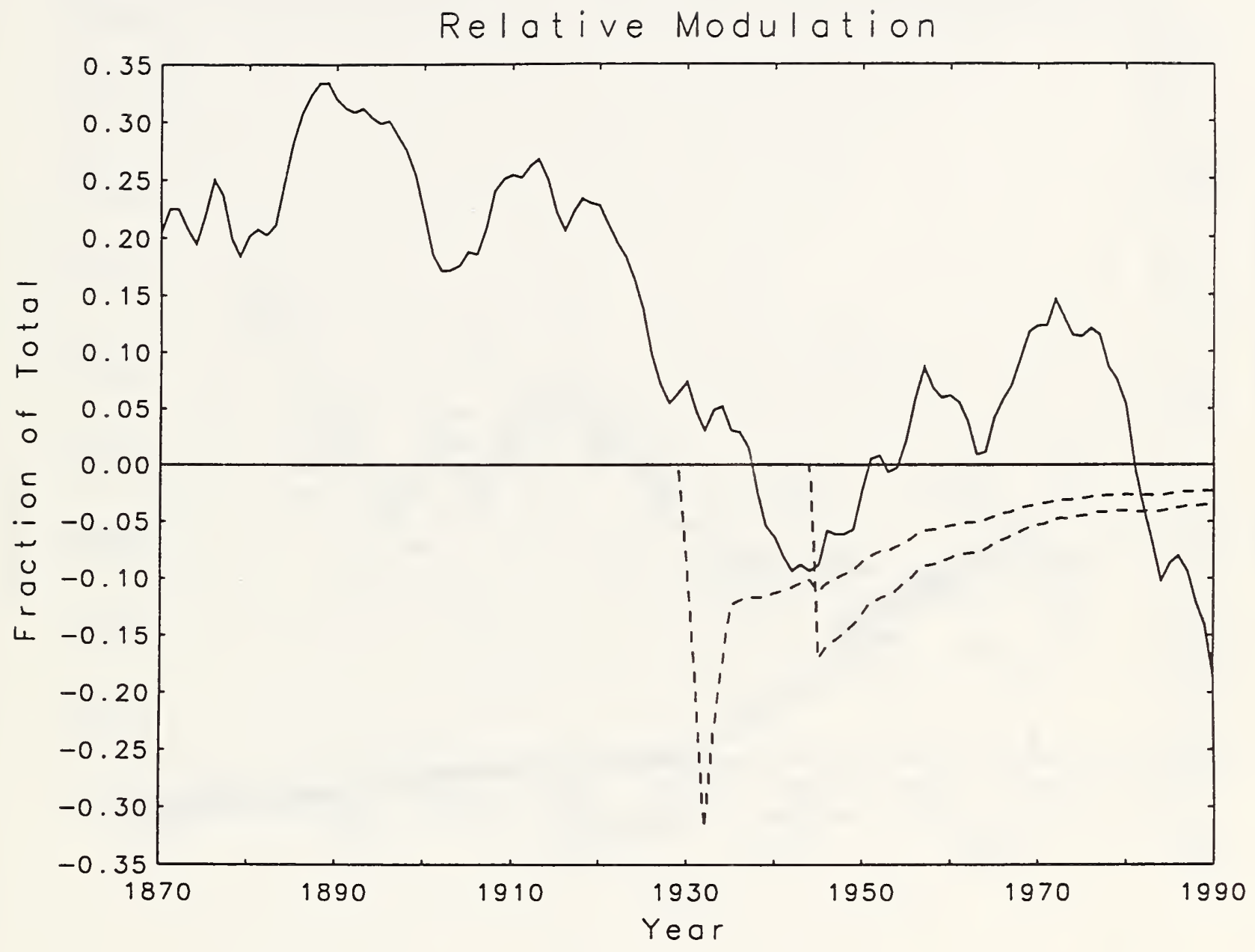

Figure 24: 
North. Hemisph. Land Temp.

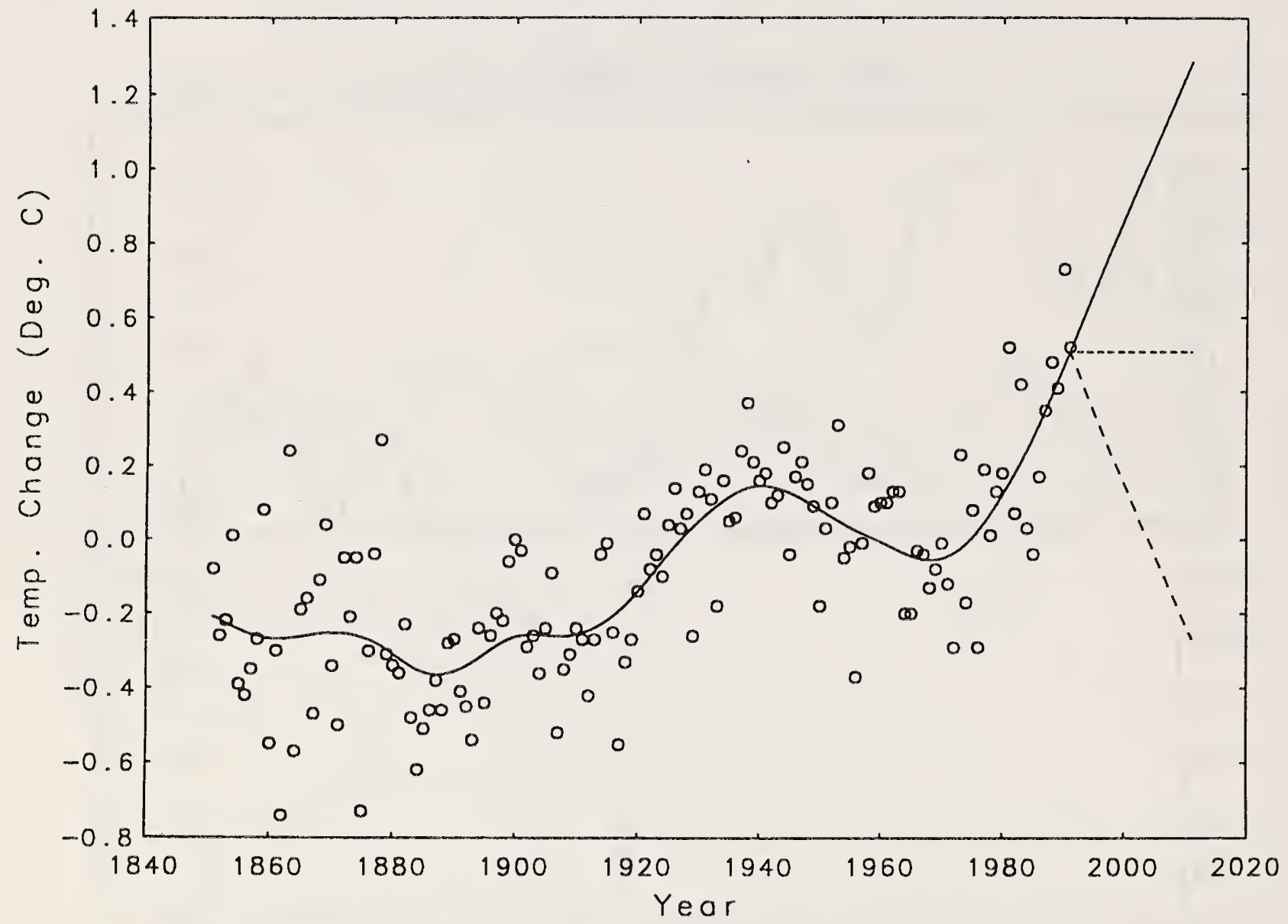

Figure 25: 


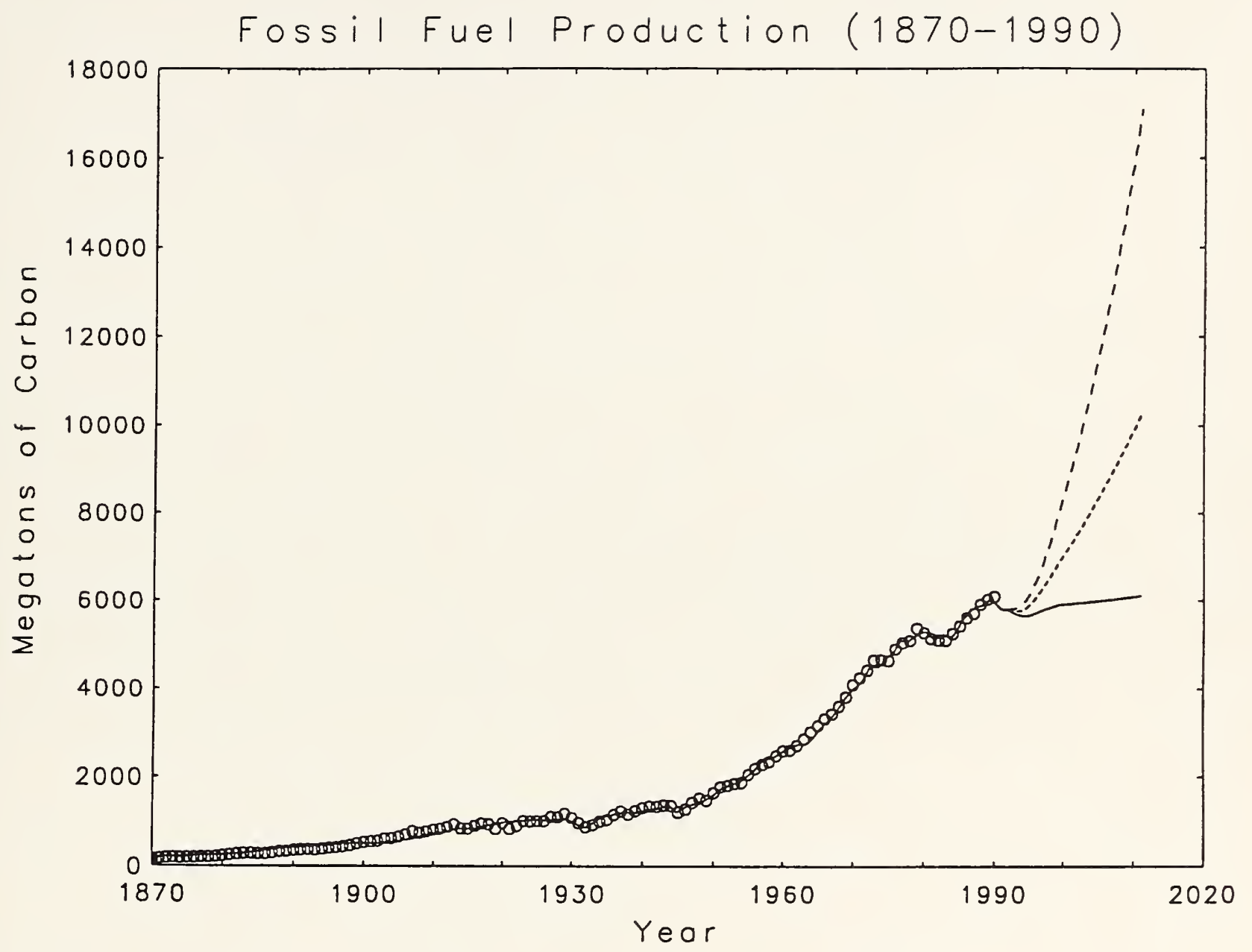

Figure 26: 

